\title{
Recent advances in small molecular modulators targeting histone deacetylase 6
}

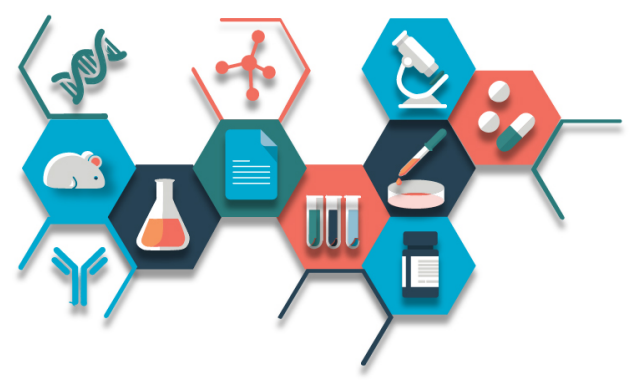

\author{
Yufeng Xiao'(iD) \& Xuan Zhang*,1(i) \\ ${ }^{1}$ Department of Medicinal Chemistry, College of Pharmacy, University of Florida, 1333 Center Drive, Gainesville, FL 32610, USA \\ *Author for correspondence: Xuan.zhang@cop.ufl.edu
}

\begin{abstract}
Histone deacetylase 6 (HDAC6) is a unique isozyme in the HDAC family with various distinguished characters. HDAC6 is predominantly localized in the cytoplasm and has several specific nonhistone substrates, such as $\alpha$-tubulin, cortactin, Hsp90, tau and peroxiredoxins. Accumulating evidence reveals that targeting HDAC6 may serve as a promising therapeutic strategy for the treatment of cancers, neurological disorders and immune diseases, making the development of HDAC6 inhibitors particularly attractive. Recently, multitarget drug design and proteolysis targeting chimera technology have also been applied in the discovery of novel small molecular modulators targeting HDAC6. In this review, we briefly describe the structural features and biological functions of HDAC6 and discuss the recent advances in HDAC6 modulators, including selective inhibitors, chimeric inhibitors and proteolysis targeting chimeras for multiple therapeutic purposes.
\end{abstract}

First draft submitted: 8 August 2020; Accepted for publication: 9 October 2020; Published online:

26 October 2020

Keywords: acetylation $\bullet$ cancer $\bullet$ chimeric inhibitor $\bullet$ degradation $\bullet$ epigenetic $\bullet$ HDAC6 $\bullet$ HDAC inhibitor $\bullet$ multitarget drug • neurodegenerative disease $\bullet$ PROTAC

Epigenetic modifications are vital physiological processes that regulate gene expression and induce phenotype changes. The status of histone acetylation plays an essential role in altering the accessibility of gene transcription and downstream cellular functions [1,2]. Typically, histone acetylation levels are regulated by HDACs and HATs [3]. The known zinc-dependent HDACs can be divided into four groups: class I (HDACs 1-3 and 8), class IIa (HDACs 4, 5, 7 and 9), class IIb (HDACs 6 and 10) and class IV (HDAC 11), with a total number of 11 HDAC isozymes being identified to date. Those HDACs present different features that vary from subcellular localization to enzymatic and nonenzymatic activities [4].

HDAC6 belongs to the HDAC IIb family and is primarily localized in the cytoplasm. Human HDAC6 is the largest member of the zinc-dependent HDAC family with a single polypeptide chain of 1215 amino acids. Unlike other HDACs that are mainly responsible for the deacetylation of nuclear histones, HDAC6 primarily targets cytoplasmic clients, such as $\alpha$-tubulin [2], cortactin [5], Hsp90 [6,7], tau [8] and peroxiredoxins [9,10], thereby playing a vital role in cell mobility, cell survival angiogenesis, protein degradation and redox regulation [11]. Due to its diverse functions to modulate many cellular pathways, HDAC6 has been elucidated as a potential therapeutic target in several diseases such as cancer, neurodegenerative diseases and immune disorders [12]. On one hand, HDAC6 regulates the acetylation of essential nonhistone proteins that are involved in cancer-relevant biological pathways such as cell migration, metastasis, angiogenesis and stress response [11]. On the other hand, HDAC6 is capable of binding the misfolded proteins to dynein motors, resulting in the transportation of ubiquitinated aggregates to the aggresome compartment, which is a crucial pathway to attenuate misfolded protein-induced stress [13,14]. One common feature of neurodegenerative dysfunction is associated with abnormal accumulation of misfolded proteins [15]. Notably, the ablation of many HDACs is lethal in mice or leads to severe physiological dysfunction. In contrast, HDAC6 knockout mice are viable and develop normally [16], suggesting that selective inhibition or even degradation of HDAC6 might be well tolerated. 
Over the last few decades, four HDAC inhibitors (HDACi): vorinostat (SAHA), romidepsin (FK228), panobinostat (LBH-589) and belinostat (PXD101) have been approved by US FDA for the treatment of hematologic cancers, either as a single agent or in combination with other drugs. Despite the FDA's approval, all these HDACi are pan-HDAC inhibitors and have limited therapeutic outcomes with solid tumors. The exact reasons are not well understood, but some observations suggest that the clinically used pan-HDAC drugs cannot sufficiently reach the effective concentration in solid tumors, leading to inadequate blockage of HDACs. Moreover, nonselective inhibition of HDACs causes various adverse effects and toxicities, such as diarrhea, fatigue, neutropenia, neurotoxicity, bone marrow toxicity, thrombocytopenia, anemia and cardiotoxicity [17-20]. Many shreds of evidence have demonstrated that selectively targeting HDAC6 might have therapeutic potential for various diseases [21,22], including cancer, neurodegenerative diseases [23,24], kidney diseases [25] and autoimmune disorders [26,27], while alleviating side effects by sparing other off-target HDAC isozymes. Moreover, overexpressed HDAC6 levels in several diseases, such as certain types of cancer [28] and neurodegenerative diseases [29], were also observed. Extensive efforts have been made in developing HDAC6-targeting modulators. The HDAC6-specific inhibitors accounted for the majority of HDAC6 modulators [30]. The general structure of an HDACi consists of a zinc-binding group (ZBG), a spacer and a capping group (cap). Clinical attempts of using HDAC6 inhibitors are intensively focused on cancer treatment, and selective HDAC6 inhibitors, including ACY-1215 (NCT02632071, NCT01997840, NCT02787369, NCT01583283), ACY-241 (NCT02400242, NCT02635061) and KA2507 (structure not disclosed, NCT04186156), are in the clinical trials either as a single agent or in combination therapy. Besides, many efforts have been made to develop HDAC6 modulators for the treatment of neurodegenerative diseases. For instance, the inhibition of HDAC6 was proved to reduce tau phosphorylation and aggregation, leading to improved learning and memory impairments in animals [29,31-34]. To date, two HDAC6 inhibitors, CKD-504 (structure not disclosed, NCT03713892) and CKD-506 (structure not disclosed, NCT04204603), have entered Phase I clinical trial for the treatment of Huntington's disease [29]. Given the great success of combination therapies in the clinic, multi-target drug (MTD) design is emerging as another effective strategy to enhance potency and overcome drug resistance [35]. The structural amenability of HDACi, especially in the cap, makes it feasible to develop MTDs with optimal potency and selectivity. Recently, proteolysis targeting chimera (PROTAC) has become a revolutionary technology in drug discovery $[36,37]$. Several examples in the literature have demonstrated that HDAC6 could be selectively degraded by converting either HDAC6-specific or even pan-HDAC inhibitors into PROTACs [38-42]. Taken together, selective antagonists, chimeric inhibitors and PROTAC degraders represent three different approaches targeting HDAC6 that exert potential therapeutic value.

\section{Structural features of HDAC6}

Structurally, unlike other HDACs that only have one catalytic domain (CD), HDAC6 contains two different CDs, namely CD1 and CD2 (Figure 1A), as well as a unique zinc-finger ubiquitin-binding domain (ZnF-UBD) [43,44]. While CD2 of HDAC6 mainly functions as $\alpha$-tubulin and tau deacetylase, the substrate specificity and function of CD1 remain less understood and CD1 exhibits much narrower substrate specificity compared with CD2 [45]. Recently, several co-crystal structures of CD1 with pan-HDAC inhibitors were disclosed, giving structural insights on the difference between CD1 and CD2 [46]. It appeared that the active site of HDAC6 CD1 is wider than that of CD2. However, most efforts were focused exclusively on targeting CD2 during the development of HDAC6-selective inhibitors. The crystal structure of human HDAC6 CD2 domain with trichostatin A (TSA) has been fully solved (PDB: 5EDU), revealing the binding pocket of $\mathrm{CD} 2$ exhibits certain structural differences in comparison to other HDACs [45], enabling the structure-based design of HDAC6-selective inhibitors. The catalytic $\mathrm{Zn}^{2+}$ in the CD2 binding pocket always coordinates with $\mathrm{ZBG}$, and the hydroxamate group seems favorable to achieve higher potency by two different coordination geometries wherein unique monodentate zinc coordination was observed by coordinating to $\mathrm{Zn}^{2+}$ only through the $\mathrm{OH}$ group without displacing the $\mathrm{Zn}^{2+}$-bound water molecule $[47,48]$. Although there are concerns related to the low solubility, poor pharmacokinetic properties and potential genotoxicity [49,50], hydroxamates still stand out as the most widely used ZBG in HDAC6-selective inhibitors. For the linker part, an aromatic ring is usually immediately adjunct to the ZBG, leading to the favorable engagement of offset $\pi-\pi$ stacking interactions with aromatic residues F583 and F643 in CD2 [51]. Besides, saturated and unsaturated aliphatic linkers have also been employed in HDAC6 CD2 binders. Appreciable HDAC6 selectivity can be achieved in case the linker length is appropriately incorporated with the ZBG, and it usually ranges between ca. 3 and $7 \AA$ [48]. As ZBG and linker regions have a limited contribution to HDAC6 selectivity, the cap is the 

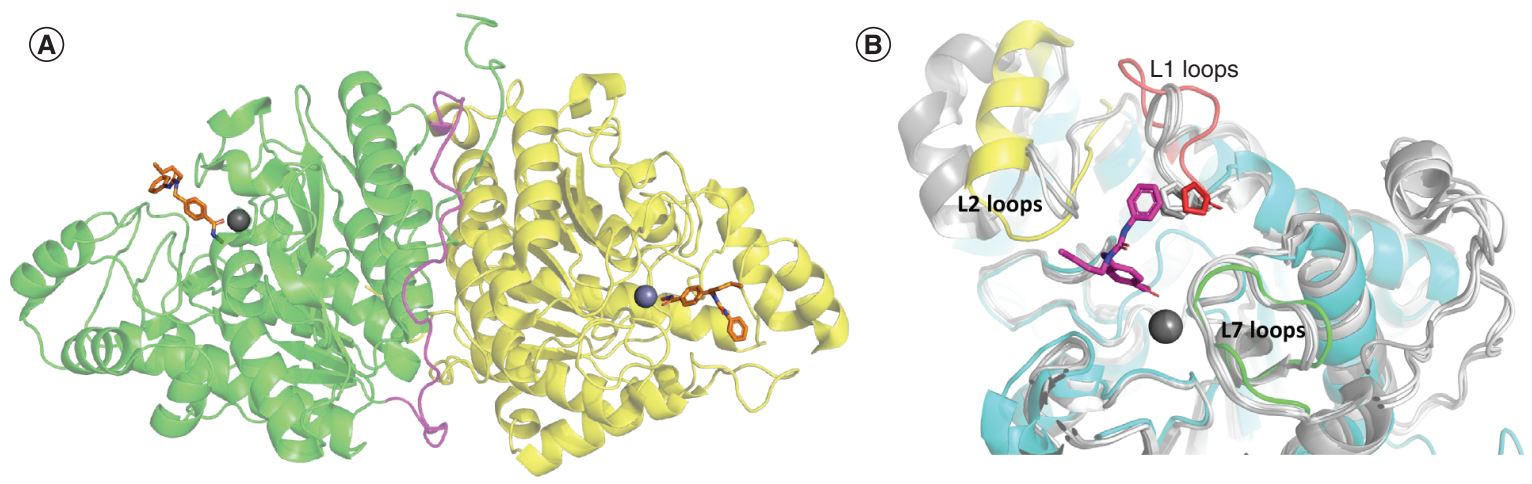

Figure 1. Structural features of histone deacetylase 6 CD1 and CD2. (A) X-ray crystal structure of NextA (orange) bound to Danio rerio HDAC6 (drHDAC6) CD1 (yellow) and CD2 (green). Zinc ions are shown as gray spheres and the interdomain linker is shown in magentas (PDB ID: 5G0I and 5G0J). (B) Superposition of HDAC6 CD2 (cyan) complexed with NextA (magentas), HDAC1 (PDB ID: 4BKX), HDAC2 (PDB ID: 4LY1) and HDAC3 (PDB ID: 4A69). All class I HDACs are shown in shades of gray. The L1, L2 and L7 loops of HDAC6 are shown in shades of red, yellow and green, respectively. The conserved proline residue is shown on L1 loops to highlight the structural differences of HDAC6 compared with class I HDACs.

most diverse part of HDAC6-specific antagonists. Many modifications have been investigated to fine-tune the selectivity/activity profile of HDAC6. The binding interactions of the cap with L1, L2 and L7 can contribute to affinity and selectivity (Figure 1B). To be more specific, caps can be designed to generate specific interaction with residues in HDAC6 that are absent in other HDACs. For example, S531 is unique to the HDAC6 CD2 active site, which accepts hydrogen bond interaction with selective inhibitors such as ACY-1083 [47]. Moreover, molecules that fit in the three-dimensional contour of the binding pocket also exhibit good affinity and selectivity for HDAC6, even without newly formed hydrogen bond interactions. Additionally, the L1 and L2 loops are relatively rigid, making HDAC6 an ideal receptor for in silico docking study. Some observations also revealed that the pan-inhibitors usually clustered around a 'hot spot' on the L1 loop by interacting with H463 and P464, whereas the contact with L2 loop might favor the selective inhibition of HDAC6 [52]. Finally, targeting the ZnF-UBD of HDAC6 provides a unique method to regulate HDAC6-mediated aggresome formation and lysosomal degradation [53]. Several inhibitors targeting ZnF-UBD have been successfully discovered [54,55].

\section{HDAC6-selective inhibitors}

The development of HDAC6-specific inhibitors is the most practical strategy to block HDAC6 activity. To date, many selective HDAC6 inhibitors have been reported, and some of them have been summarized in previous review articles $[11,29,56]$. Here we present the representative compounds in those reviews and focus on the new compounds (2016-2020) with high potency (typically HDAC6 $\mathrm{IC}_{50}<1 \mu \mathrm{M}$ ), decent selectivity (HDACs/HDAC6 > 10) and at least three available binding affinity data of other HDAC isoforms (HDAC1, 2, 3, 6 and 8). Notably, since $\alpha$-tubulin is a specific substrate for HDAC6 but not other HDACs, the selective inhibition of HDAC6 can be characterized by hyperacetylation of tubulin (biomarker: Ac- $\alpha$-tubulin) without affecting the level of histone acetylation (biomarker: Ac-histone H3).

\section{Fatty hydroxamic acid-derived HDAC6 inhibitors}

The chemical structures of fatty hydroxamic acid-derived HDAC6 inhibitors are depicted in Figure 2. Tubacin (1) was discovered in the early years by a chemical-genetic screen of 7392 small molecules [57]. It was found to inhibit $\alpha$-tubulin deacetylation in mammalian cells without affecting the level of histone acetylation, geneexpression patterns or cell-cycle progression. The binding affinity of tubacin toward HDAC6 was around $4 \mathrm{nM}$, which was 350-fold more selective over HDAC1 [58]. The treatment of tubacin resulted in the alleviation of tau phosphorylation, suggesting its therapeutic potential for Alzheimer's disease (AD) [59]. In addition, it also showed that tubacin treatment triggered apoptotic pathways in pre-B and $\mathrm{T}$ cell acute lymphoblastic leukemia cells with nanomolar-range activity, and the combination with other chemotherapeutic drugs further enhanced the potency in acute lymphoblastic leukemia cells both in vitro and in vivo [60]. Tubacin is widely used as a tool compound in 

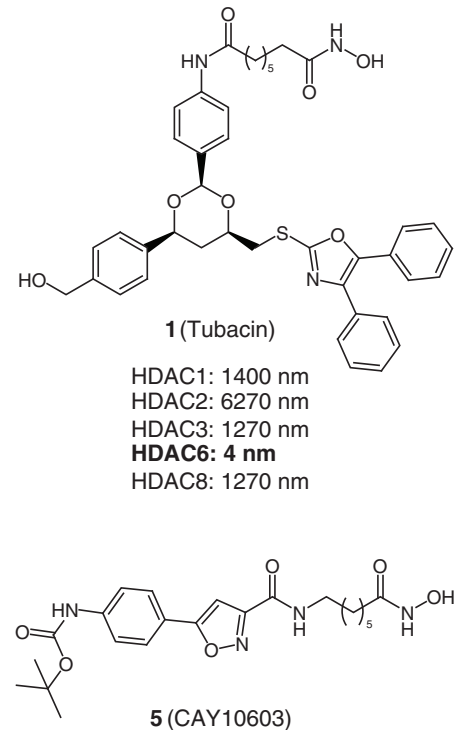

HDAC1: $271 \mathrm{~nm}$ HDAC2: $252 \mathrm{~nm}$ HDAC3: $0.42 \mathrm{~nm}$ HDAC6: $0.002 \mathrm{~nm}$ HDAC8: NA

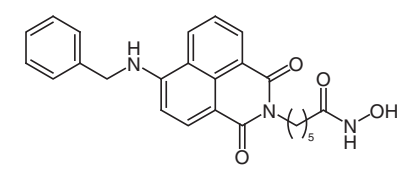

9

HDAC1: $1980 \mathrm{~nm}$ HDAC2: ND HDAC3: $360 \mathrm{~nm}$ HDAC6: $3.5 \mathrm{~nm}$ HDAC8: $2460 \mathrm{~nm}$<smiles>O=C(CCCC(=O)N/N=C/c1ccc(N(c2ccccc2)c2ccccc2)cc1)NO</smiles>

2 (WT161)

HDAC1: $8.35 \mathrm{~nm}$ HDAC2: $15.4 \mathrm{~nm}$ HDAC3: $51.6 \mathrm{~nm}$ HDAC6: $0.4 \mathrm{~nm}$ HDAC8: $1430 \mathrm{~nm}$

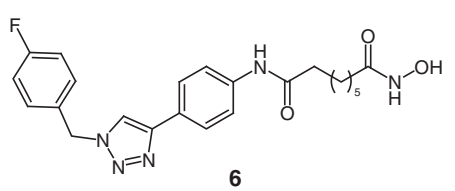

HDAC1: $97.8 \mathrm{~nm}$ HDAC2: $294 \mathrm{~nm}$ HDAC3: $13.7 \mathrm{~nm}$ HDAC6: $1.9 \mathrm{~nm}$ HDAC8: $797 \mathrm{~nm}$<smiles>Cc1nc2cc(/C=C/C(=O)NO)ccc2c(=O)n1CCc1ccccc1</smiles>

10

HDAC1: $1880 \mathrm{~nm}$ HDAC2: $6450 \mathrm{~nm}$ HDAC3: ND HDAC6: $29 \mathrm{~nm}$ HDAC8: $1750 \mathrm{~nm}$

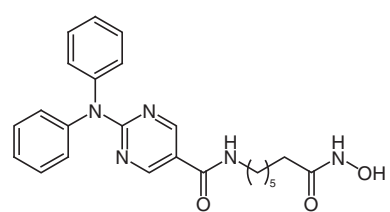

3 (ACY-1215, ricolinostat)

HDAC1: $58 \mathrm{~nm}$ HDAC2: $48 \mathrm{~nm}$ HDAC3: $51 \mathrm{~nm}$ HDAC6: $4.7 \mathrm{~nm}$ HDAC8: $100 \mathrm{~nm}$

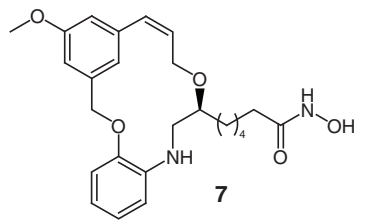

HDAC1: $32 \mathrm{~nm}$ HDAC2: $158 \mathrm{~nm}$ HDAC3: $55.7 \mathrm{~nm}$ HDAC6: $0.84 \mathrm{~nm}$ HDAC8: $198 \mathrm{~nm}$

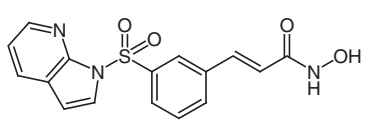

11

HDAC1: $310 \mathrm{~nm}$ HDAC2: $1160 \mathrm{~nm}$ HDAC3: $>10,000 \mathrm{~nm}$ HDAC6: $5.2 \mathrm{~nm}$ HDAC8: $1390 \mathrm{~nm}$<smiles></smiles>

4 (ACY-241, citarinostat) HDAC1: $35 \mathrm{~nm}$ HDAC2: $45 \mathrm{~nm}$ HDAC3: $46 \mathrm{~nm}$ HDAC6: $2.6 \mathrm{~nm}$ HDAC8: $137 \mathrm{~nm}$<smiles>COc1ccc(N(C)c2nc(C)nc3ccccc23)cc1OCCCC(=O)NO</smiles>

HDAC1: $422 \mathrm{~nm}$ HDAC2: $386 \mathrm{~nm}$ HDAC3: $439 \mathrm{~nm}$ HDAC6: $17 \mathrm{~nm}$ HDAC8: $3398 \mathrm{~nm}$

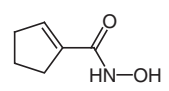

12 (BRD9757)

HDAC1: $638 \mathrm{~nm}$ HDAC2: $1790 \mathrm{~nm}$ HDAC3: $694 \mathrm{~nm}$ HDAC6: $30 \mathrm{~nm}$ HDAC8: $1090 \mathrm{~nm}$

Figure 2. The structures of fatty hydroxamic acid-derived histone deacetylase 6 inhibitors.

HDAC6: Histone deacetylase 6; NA: Not active; ND: Not determined.

many in vitro studies. However, the poor pharmacokinetics (PK) properties and structural complexity of tubacin limit its further development.

To simplify the structure of tubacin, Hideshima et al. built a small compound library and identified WT161 (2) as a potent HDAC6 antagonist $\left(\mathrm{IC}_{50}=0.4 \mathrm{nM}\right)$ with remarkable selectivity over $\mathrm{HDAC} 1,2,3$ and 8 . In addition, favorable PK profile such as reasonable half-life in mice $\left(\mathrm{t}_{1 / 2}=1.4 \mathrm{~h}\right)$ and adequate drug exposure assured the in vivo efficacy [61]. ACY-1215 (3, ricolinostat) was another well-studied HDAC6 inhibitor with an $\mathrm{IC}_{50}$ of $4.7 \mathrm{nM}$ and at least tenfold selectivity over HDAC 1-3 [53]. As an orally bioavailable drug candidate, it entered clinical trial for the treatments of malignancies as well as diabetic neuropathic pain [62]. Meanwhile, ACY-241 (4) was developed as the second generation of ACY-1215 with improved solubility. It exhibited an $\mathrm{IC}_{50}$ value of $2.6 \mathrm{nM}$ against HDAC6 with moderate selectivity over other HDACs. ACY-241 is now in Phase I clinical trial as a combination therapy for the advanced solid tumors and multiple myeloma treatments [63].

Kozikowski et al. reported a series of HDAC6 inhibitors bearing phenylisoxazole caps. Among them, compound 5 showed extremely high activity against HDAC6 $\left(\mathrm{IC}_{50}<2 \mathrm{pM}\right)$ along with superior selectivity over HDAC1, 2 , 3,8 and 10 (more than 200-fold). In vitro study validated its cell-killing effect in several pancreatic cancer cell lines, and the potency was higher than the pan-HDAC inhibitor SAHA (vorinostat) [64]. Chen and co-workers developed a series of triazolylphenyl-based HDACi and found that the substitution on the phenyl ring affected the selectivity for HDAC6 versus HDAC1, among which the p-fluorinated phenyl derivative $\mathbf{6}$ gave the best HDAC6 selectivity over HDAC1 while maintaining high activity against HDAC6 $\left(\mathrm{IC}_{50}=1.9 \mathrm{nM}\right)$ [65]. Macrocyclic inhibitors such as apicidin $\mathrm{A}$ and trapoxin $\mathrm{B}$ represent another type of $\mathrm{HDACi}$, and most of them are natural products with 
limited isozyme selectivity. Auzzas et al. reported a new class of non-natural macrocyclic HDACi, and their binding profile against all HDAC isoforms was evaluated. Highly conserved residues on the binding surface among HDAC isozymes were taken into account in the design, which led to the discovery of compound 7, a potent HDAC6 inhibitor $\left(\mathrm{IC}_{50}=0.84 \mathrm{nM}\right)$ with moderate isoform selectivity. In contrast, the $(R)$-enantiomer of 7 showed a 12-fold decrease in binding affinity for HDAC6 $\left(\mathrm{IC}_{50}=10.2 \mathrm{nM}\right)$. Binding mode analysis of both enantiomers of 7 along with apicidin A led to the definition of general pharmacophoric models, which might provide valuable structural insights into the design of new HDACi [66]. For a similar purpose of creating specific interaction in the capping region, Yang et al. performed molecular docking studies and discovered a new HDAC6 inhibitor using quinazoline as the cap. In the docking study, quinazoline was capable of forming $\pi-\pi$ stacking interaction with residues Phe136 and Phe82, as well as hydrophobic interaction with residues of Ile85, Met198 and Gly135. In vitro, compound 8 showed decent binding affinity against HDAC6 $\left(\mathrm{IC}_{50}=17 \mathrm{nM}\right)$ and high HDAC6 selectivity over all other HDAC isozymes. Moreover, compound 8 presented significant antiproliferative activities in 14 tested cancer cell lines ( $\mathrm{IC}_{50}$ range: 14-104 nM). In vivo, compared with SAHA or ACY-1215, compound 8 showed comparable or improved antitumor activity in several animal models bearing HCT116, MV4-11 and Ramos xenografts. The in vivo efficacy and ideal $\mathrm{PK}$ parameters $(\mathrm{F}=47 \%)$ make it a potent oral anticancer drug candidate [67].

Fleming et al. reported fluorescent scriptaid derivatives as novel HDAC6-specific binders. Compound 9 had high potency affinity of HDAC6 $\left(\mathrm{IC}_{50}=3.5 \mathrm{nM}\right)$, outstanding selectivity $(>100-7000$ fold over other HDACs), along with ideal fluorescent parameters, warranting its application as a useful HDAC6-specific probe for in vitro cellular imaging and in vivo zebrafish imaging [68].

Yu et al. developed hydroxamic acid-based quinazolin-4-one derivatives as HDAC6-specific inhibitors. Structureactivity relationship study revealed the most promising compound $\mathbf{1 0}$ preferentially inhibited HDAC6 with an $\mathrm{IC}_{50}$ of $29 \mathrm{nM}$. In vitro, compound $\mathbf{1 0}$ induced substantial neurite outgrowth, enhanced the synaptic activities of PC12 and SH-SY5Y neuronal cells, and attenuated anti-zinc-mediated $\beta$-amyloid $(\mathrm{A} \beta)$ aggregation. In vivo, 10 alleviated $A \beta$-mediated neurodegeneration and induced learning enhancements in hippocampus-lesioned mice. Moreover, compound 10 displayed a good safety profile in the CYP450 and hERG inhibitory assessments, making it a promising lead compound for the treatment of $\mathrm{AD}[69]$.

Lee and colleagues prepared a series of indolylsulfonylcinnamic hydroxamates as HDAC6 inhibitors [70]. Compound 11, a potent HDAC6 antagonist with an $\mathrm{IC}_{50}$ of $5.2 \mathrm{nM}$, showed comparable antiproliferative activity as SAHA in KB, H146, PC3 and HCT116 cancer cell lines. This compound had a good PK profile with an oral bioavailability of $33 \%$ and suppressed tumor growth in colorectal HCT116 xenografts model $(100 \mathrm{mg} / \mathrm{kg}$, p.o., q.d.; TGI $=66.9 \%$ ). Interestingly, Wagner and colleagues reported 12 (BRD9757), a capless HDAC6 inhibitor with excellent potency $\left(\mathrm{IC}_{50}=30 \mathrm{nM}\right)$ and decent selectivity toward HDAC6 versus HDAC1-9. Upon treatment of $\mathbf{1 2}$ in a cell-based assay, selective increase in the level of Ac-tubulin but not histone acetylation was observed, confirming that potent and selective inhibition of HDAC6 can be achieved through careful choice of linker element [71].

\section{Aromatic hydroxamic acid-derived inhibitors}

The chemical structures of aromatic hydroxamic acid-derived HDAC6 inhibitors are illustrated in Figure 3. Most of them are derivatives of phenylhydroxamate. De Vreese and colleagues built a small library of benzothiophenebased hydroxamic acids as HDAC6 inhibitors [72]. Compound $\mathbf{1 3}$ stood out in this library for its excellent HDAC6 activity/selectivity profile $\left(\mathrm{IC}_{50}=14 \mathrm{nM}\right)$. Further study demonstrated that HDAC6 inhibition could be uncoupled from transcriptional inhibition at the level of activated NF- $\mathrm{kB}, \mathrm{AP}-1$ and GR, although further in-depth studies are required.

Lee and colleagues developed ( $N$-hydroxycarbonylbenylamino) quinolines as selective HDAC6 antagonists. Compound 14 showed remarkable HDAC6 inhibition with an $\mathrm{IC}_{50}$ of $0.29 \mathrm{nM}$, which is at least 1200-times more selective than other HDAC isozymes. Moreover, it displayed antiproliferative activity against human multiple myeloma RPMI 8226, U266 and NCI-H929 cells while sparing normal bone marrow cells. In the hepatocyte stability experiment, compound $\mathbf{1 4}$ exhibited an acceptable half-life and clearance rate, especially in human species. The antimutagenic safety profile was confirmed by the Ames test. In vivo study demonstrated that 14, either alone or in combination with bortezomib, suppressed tumor growth in human multiple myeloma xenograft models, and all model mice in the combination group survived until the end of the experiment, indicating the potential of $\mathbf{1 4}$ to be developed as a promising agent for the treatment of multiple myeloma [73]. 
<smiles>NC(=O)c1ccc(NCc2csc3ccccc23)cc1</smiles>

13

HDAC1: $7500 \mathrm{~nm}$

HDAC2: $30,000 \mathrm{~nm}$

HDAC6: $14 \mathrm{~nm}$

HDAC8: $1400 \mathrm{~nm}$

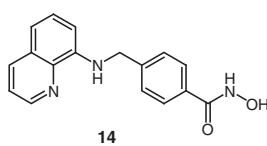

14

HDAC1: $9550 \mathrm{~nm}$ HDAC2: $12,500 \mathrm{~nm}$ HDAC6: $0.291 \mathrm{~nm}$ HDAC8: $1190 \mathrm{~nm}$<smiles>O=C(NO)c1ccc(CN2c3ccccc3NC(=O)C2Cc2ccc(O)cc2)cc1</smiles>

19

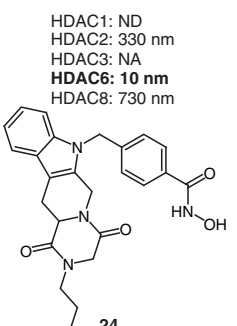

HDAC1: $8450 \mathrm{~nm}$ HDAC2: $3340 \mathrm{~nm}$ HDAC3: NA HDAC6: $3.73 \mathrm{~nm}$
HDAC8: $747 \mathrm{~nm}$

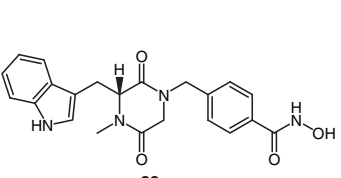
29 HDAC1: $8020 \mathrm{~nm}$ HDAC2: NA HDAC3: NA HDAC8: $513 \mathrm{~nm}$<smiles></smiles>

20 HDAC1: $1710 \mathrm{~nm}$ HDAC3: NA HDAC6: $12.4 \mathrm{~nm}$
HDAC8: $160 \mathrm{~nm}$

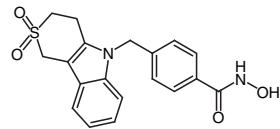

25

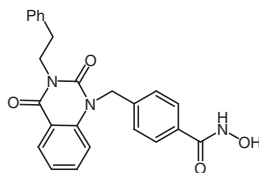

21 (J22352) HDAC1: $11,160 \mathrm{~nm}$ HDAC2: $>10,000 \mathrm{~nm}$ HDAC3: $>10,000 \mathrm{~nm}$ HDAC6: $4.7 \mathrm{~nm}$ HDAC8: $376.2 \mathrm{~nm}$

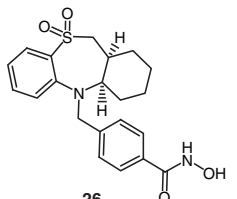

HDAC1: $11,000 \mathrm{~nm}$ HDAC2: $26,000 \mathrm{~mm}$ HDAC3: $29,000 \mathrm{~nm}$
HDACC: $19 \mathrm{~nm}$ HDAC8: $17,000 \mathrm{~nm}$ Cl
HDAC1: $8100 \mathrm{~nm}$ HDAC2: $24,000 \mathrm{~nm}$ HDAC3: $18,000 \mathrm{~nm}$ HDAC6: $8 \mathrm{~nm}$

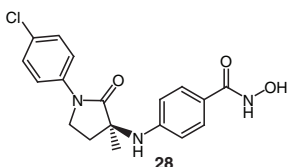

HDAC1: $741000 \mathrm{~nm}$ HDAC2: $>10,000 \mathrm{~nm}$ HDAC6: $17 \mathrm{~nm}$ HDAC8: $180 \mathrm{~nm}$

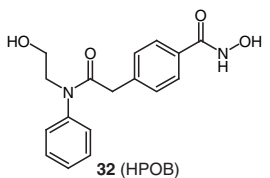

HDAC1: $2900 \mathrm{~nm}$ HDAC2: $4400 \mathrm{~nm}$ HDAC6: $56 \mathrm{~nm}$ HDAC8: $2800 \mathrm{~nm}$

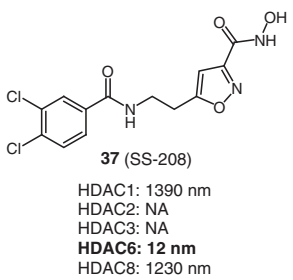

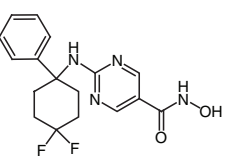

33 (ACY-1083) HDAC6: $3 \mathrm{~nm}$ over other HDAC

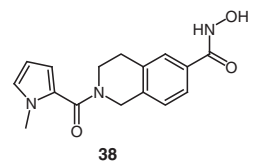

HDAC1: $45,000 \mathrm{~nm}$ HDAC3: $46,000 \mathrm{~nm}$ HDAC6: $36 \mathrm{~nm}$ HDAC8: $2100 \mathrm{~nm}$

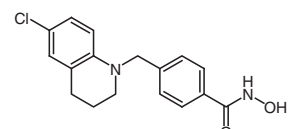

$15(\mathrm{SW}-100)$ HDAC1: $5230 \mathrm{~nm}$ HDAC6: $23.30 \mathrm{~mm}$ HDAC8: $3720 \mathrm{~nm}$ HDAC2: $32,800 \mathrm{~nm}$

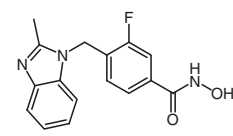

16

HDAC1: $8960 \mathrm{~nm}$ HDAC2: $20,300 \mathrm{n}$ HDAC3: $12,500 \mathrm{~nm}$ HDAC8: $176 \mathrm{~nm}$

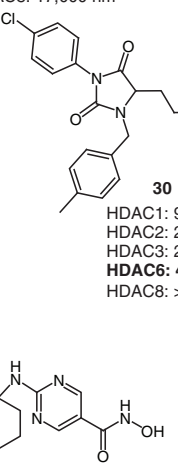

34 (ACY-775) HDAC1: $2123 \mathrm{~nm}$ HDAC3: $11.223 \mathrm{~nm}$ HDAC6: $7.5 \mathrm{~nm}$ HDAC8: NA

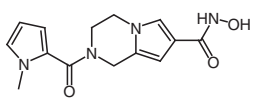

39

HDAC1: $15,000 \mathrm{~nm}$ HDAC2: $39,000 \mathrm{~nm}$ HDAC6: $33 \mathrm{~nm}$ HDAC8: $3300 \mathrm{~nm}$

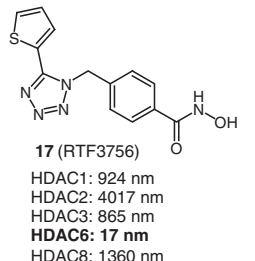

HDAC8: $1360 \mathrm{~nm}$

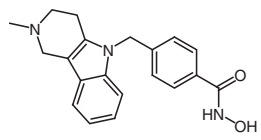

22 (Tubastatin A, TuA)

HDAC1: $16,400 \mathrm{~nm}$ HDAC2: $>30,000 \mathrm{~nm}$ HDAC3: >30,000 nm HDAC6: $15 \mathrm{~nm}$

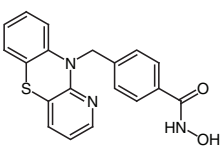

27

HDAC1: $2690 \mathrm{~nm}$ HDAC2: ND HDAC3: ND HDAC8: $3100 \mathrm{~nm}$<smiles>CCCCN(Cc1ccc(C(=O)NO)cc1)C(=O)Nc1ccccc1</smiles>
HDAC1: $3020 \mathrm{~nm}$ HDAC3: $6280 \mathrm{~nm}$ HDAC6: $5.02 \mathrm{~nm}$ HDAC8: $954 \mathrm{~nm}$

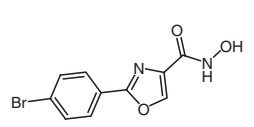

36

HDAC1: $14,000 \mathrm{~nm}$ HDAC3: NA HDAC6: $59 \mathrm{~nm}$ HDAC8: $14,000 \mathrm{~nm}$ HDAC8: NA

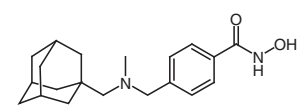

41

HDAC1: $>10,000 \mathrm{~nm}$ DAC3: $>10,000 \mathrm{~nm}$ HDAC6: $60 \mathrm{~nm}$ HDAC8: $8500 \mathrm{~nm}$

Figure 3. The structures of aromatic hydroxamic acid-derived inhibitors. HDAC6: Histone deacetylase 6; NA: Not active; ND: Not determined.

Kozikowski et al. reported a unique brain penetrable HDAC6 Inhibitor SW-100 (15). This compound displayed a low nanomolar activity against HDAC6 with an excellent $>1500$-fold reduced potency against all other zincdependent HDACs. At the cellular level, SW-100 met the hallmark of HDAC6 CD2 inhibition to increase the acetylation level of $\alpha$-tubulin in a dose-dependent manner without affecting the histone acetylation in HEK293 cells. SW-100 had ideal brain bioavailability $(141.8 \mathrm{ng} / \mathrm{ml}$ in brain, i.p. $20 \mathrm{mg} / \mathrm{kg}$ ), and showed promising efficacy in the improvement of memory performance in a mouse model of FXS, $\mathrm{Fmr}^{-1-}$ mice. Moreover, the ADME (absorption, 
distribution, metabolism and excretion) evaluation revealed that SW-100 had acceptable hERG-inhibitory activity and liver microsomal stability, and showed negative results in the Ames test [74].

Shen and coworkers discovered compound $\mathbf{1 6}$ through structural modifications [75]. Compound $\mathbf{1 6}$ not only displayed higher potency against HDAC6 $\left(\mathrm{IC}_{50}=0.81 \mathrm{nM}\right)$ and impressive selectivity over other HDACs but also exhibited improved PK $(\mathrm{F}=20.9 \%)$ in comparison to TubA $(\mathrm{F}=5.88 \%)$. Docking study suggested that in addition to the $\pi-\pi$ stacking between benzimidazole and Phe680 on the cavity rim, the $N^{3}$ nitrogen could elicit a weak H-bond with Ser568. Charcot-Marie-Tooth disease (CMT) is an inherited disease of the peripheral nervous system. In a mutant HSPB1-induced in vitro CMT2 model, 16 was capable of restoring mitochondrial axonal transport defects, suggesting that compound $\mathbf{1 6}$ might serve as a useful lead compound for future animal studies using CMT diseases models.

Vergani and co-workers reported a novel selective HDAC6 inhibitor $\mathbf{1 7}$ bearing a tetrazole central core [76]. Remarkably, compound $\mathbf{1 7}$ had low toxicity both in vitro and in vivo, confirming selective inhibition of HDAC6 could be well tolerated by healthy cells. Moreover, 17 can increase the function of regulatory $\mathrm{T}$ cells (Tregs). Compound $\mathbf{1 7}$ is orally bioavailable and presents acceptable PK, indicating the potential to be a drug candidate for the treatment of several pathologies, including degenerative and autoimmune diseases.

While most selective HDAC6 is consist of a large and rigid cap, Gawel and colleagues discovered a novel HDAC6 inhibitor 18 (PTG-0861) bearing a pentafluorobenzene cap [77]. PTG-0861 showed low nanomolar potency $\left(\mathrm{IC}_{50}=5.92 \mathrm{nM}\right.$ ) against HDAC6 and good HDAC6-selectivity ( $>36$ folds). According to the docking study, there were potential $\pi-\pi$ stacking interactions between His500 and the electron-deficient cap. Moreover, PTG-0861 displayed antiproliferative in several cancer cell lines while showing limited cytotoxicity against normal cells. Besides, PTG-0861 showed a promising safety profile and in vitro PK.

Compound 19 with a distinct chiral capping motif showed more than 40-fold selectivity for HDAC6 over other HDAC2 and HDAC8. The observed HDAC selectivity and potency were dependent on the absolute configuration of the chiral center [78]. In another paper, Liu et al. found pyrimidinedione-derived compound 20 exhibited selective inhibition on HDAC6 $\left(\mathrm{IC}_{50}=12.4 \mathrm{nM}\right)$ over HDAC1 $\left(\mathrm{IC}_{50}=1710 \mathrm{nM}\right)$ and HDAC2 $\left(\mathrm{IC}_{50}=5500 \mathrm{nM}\right)$ [79]. Compound 20 also efficiently inhibited tumor growth in a HCT116 xenograft tumor model without overt signs of toxicity. Later on, by fine-tuning the cap, compound $\mathbf{2 1}$ (J22352) was reported as a highly selective HDAC6 inhibitor $\left(\mathrm{IC}_{50}=4.7 \mathrm{nM}\right)$. Of note, 21 showed PROTAC-like degradation activity of HDAC6, which can be blocked with proteasome inhibitor MG132. Since HDAC6 is overexpressed in glioblastoma, 21 was tested in glioma cells and found to significantly inhibit proliferation in a dose-dependent manner. Notably, $\mathbf{2 1}$ was also capable of reducing the immunosuppressive activity of PD-L1, leading to the restoration of host antitumor activity [80].

Tubastatin A (TubA, 22) is a well-known HDAC6 inhibitor $\left(\mathrm{IC}_{50}=15 \mathrm{nM}\right)$ with a bulky substitution at the benzylic position. It showed superior selectivity over HDAC1 (>3000-fold) and was initially disclosed for its neuroprotective effect. It was developed using structure-based drug design along with homology modeling techniques [58]. In vitro, TubA selectively increased the acetylation levels of $\alpha$-tubulin, but not histone. Besides, TubA and its analogs were also reported to have anti-inflammatory effects through enhancing the immunosuppressive activity of Foxp3 ${ }^{+}$Tregs [26], as well as anti-rheumatic [81] and anti-hepatitis $\mathrm{C}$ viral activities [82]. Recently, the crystal structure of drHDAC6-CD2/TubA complex had been solved, giving the molecular basis of ligand-protein interaction [83]. TubA had become a starting point for structural optimization, and several subsequent works were carried out based on its analogs.

To expand the cap of TubA, Sellmer and co-workers designed and synthesized compound $\mathbf{2 3}$ (marbostat-100) as a highly selective and potent inhibitor of HDAC6 $\left(\mathrm{IC}_{50}=0.7 \mathrm{nM}\right)$ [84]. In vitro, marbostat-100 induced tubulin acetylation while having minimal effect on histone acetylation level. Moreover, marbostat-100 (30 mg/kg) was well tolerated in mice and demonstrated anti-inflammatory activity in collagen-induced arthritis model. In a follow-up study, a series of tetracyclic tetrahydro- $\beta$-carboline derivatives were designed by Leonhardt et al. The structural modifications were focused on the carboline part, and compound $\mathbf{2 4}$ stood out among the analogs with the highest HDAC6 inhibitory activity $\left(\mathrm{IC}_{50}=3.73 \mathrm{nM}\right)$, which was fourfold more potent than TubA. In the docking study, the rigid tetracyclic surface recognizing group was well fitted into the entrance of the binding pocket. At the cellular level, 24 induced rapid tubulin hyperacetylation, even at a concentration of $50 \mathrm{nM}$ without effecting the histone acetylation [85].

The sulfur-containing functional group is widely employed in medicinal chemistry to achieve different biological activities. To date, several TubA derivatives bearing sulfone groups were developed as HDAC6 inhibitors. Systematic 
explorations on TubA, including nonaromatic ring size, oxidation state and position of sulfur, led to the discovery of compound 25 with pronounced activity $\left(\mathrm{IC}_{50}=1.9 \mathrm{nM}\right)$ and selectivity toward HDAC6 [86]. The docking study suggested that the sulfone group might establish an additional hydrogen bond with the surface residue of HDAC6. In addition, sulfone analogs had better ADME profiles than sulfide compounds, suggesting sulfone might be a favorable building block in the pursuit of novel HDAC6 inhibitors. Compound $\mathbf{2 6}$ is another HDAC6 inhibitor with sulfone building block, and it is rationally designed based on TubA by functionalizing a sulfurcontaining tricyclic cap [87]. This compound also showed high potency $\left(\mathrm{IC}_{50}=8 \mathrm{nM}\right)$ and selectivity for HDAC6. Furthermore, $\mathbf{2 6}$ and its analogs were accessed in an Ames fluctuation assay and had no mutagenic effects associated with this scaffold. In another study, Vögerl and colleagues identified the phenothiazine system as a favorable cap for HDAC6-specific inhibitors [88]. Compound 27 inhibited HDAC6 with an $\mathrm{IC}_{50}$ of $5 \mathrm{nM}$ and induced tubulin acetylation in HL60 cells. Structure-activity relationship studies revealed that the introduction of a nitrogen atom into the phenothiazine scaffold resulted in improved potency and selectivity for HDAC6. The cocrystal structure of $\mathbf{2 7}$ with $\mathrm{CD} 2$ from drHDAC6 was solved, providing a wealth of information regarding the molecular insights.

The development of hydroxamate-derived HDACi with high oral bioavailability is quite challenging. Compound 28, developed by Roche (Basel, Switzerland), was one of the few compounds that successfully achieved excellent oral bioavailability $(\mathrm{F}=55 \%)$ [89]. Meantime, it possessed high HDAC6 inhibitory potency $\left(\mathrm{IC}_{50}=17 \mathrm{nM}\right)$ and 10and 4000-fold selectivity over HDAC8 and HDAC1, respectively. Aminopyrrolidinone cap was carefully optimized to balance potency/selectivity profile, lyophilized solubility, plasma stability and PK properties. The ideal drug-like property of $\mathbf{2 8}$ warrants further in vitro and in vivo functional studies.

Chen et al. used novel 2,5-diketopiperazine skeleton to develop potent and selective HDAC6 inhibitors, and compound 29 was highlighted due to its subnanomolar activity toward HDAC6 $\left(\mathrm{IC}_{50}=0.73 \mathrm{nM}\right)$. The molecular simulation demonstrated a water-mediated hydrogen bond being formed between indole nitrogen and Asp 497 in the L1 loop of HDAC6, whereas ZBG of $\mathbf{2 9}$ poorly coordinated to the catalytic zinc ion in human HDAC1. Unfortunately, this compound showed minimal antiproliferative activities against most tested cancer cell lines, which was probably due to a relatively poor permeability [90].

Liang et al. developed novel 2,4-imidazolinedione derivatives as HDAC6 isozyme-selective inhibitors through structure-based drug design [91]. Compound 30 exerted potent HDAC6 inhibitory activity $\left(\mathrm{IC}_{50}=4.4 \mathrm{nM}\right)$ and an excellent HDAC6 selectivity profile. Besides, compound $\mathbf{3 0}$ was capable of inducing apoptosis in HL-60 cells by activating caspase- 3 and significantly increase the acetylation of $\alpha$-tubulin with minimal effect on the acetylation of $\mathrm{H} 3$. Since $\mathbf{3 0}$ was synthesized as a racemic mixture, the separation of enantiomers will be required in future studies.

Bergman and co-workers designed a series of HDACi bearing urea-containing linkers [92]. Urea motif is a useful building block in drug discovery due to its strong hydrogen bonding potential with protein. While the unsubstituted urea-based compounds appeared to be nonselective, Nexturastat A (NextA, 31), a representative compound with substituted urea linker achieved dramatically increased potency $\left(\mathrm{IC}_{50}=5.02 \mathrm{nM}\right)$ and excellent selectivity for HDAC6 versus the full panel of HDACs. NextA could cause a dose-dependent increase of acetyl $\alpha$-tubulin levels on $\mathrm{B} 16$ murine melanoma cells without a concomitant elevation of histone $\mathrm{H} 3$ acetylation, indicating the specificity toward HDAC6. NextA is the first HDAC6-specific inhibitor suppressed melanoma cell growth, suggesting the possible value in oncology.

Lee $e$ al . reported that compound 32 (HPOB) could selectively inhibit HDAC6 catalytic activity with an IC $_{50}$ value of $56 \mathrm{nM}$ [93]. HPOB suppressed the growth of normal and transformed cells but did not induce cell death. Furthermore, HPOB had a synergistic effect in combination with SAHA or DNA-targeting anticancer drugs such as etoposide and doxorubicin in transformed cells but not normal cells, indicating that HDAC6 combinational treatment might have promising therapeutic values.

ACY-1083, ACY-775 and ACY-738 (33-35), developed by Acetylon Pharmaceuticals (MA, USA), all possess a pyrimidine moiety directly attached to the hydroxamic acid ZBG. ACY-775 and ACY-738 exhibited single-digit nanomolar potency against HDAC6 and decent selectivity over class I HDACs. In vivo, these two compounds are brain penetrable and showed antidepressant-like properties in mice models [94]. ACY-1083, reported by Krukowski et al., showed an $\mathrm{IC}_{50}$ of $3 \mathrm{nM}$ against HDAC6 and was 260-fold more selective over HDAC 1-9. In in vivo PK evaluation, this compound revealed a decent plasma exposure with a cmax value of $936 \mathrm{ng} / \mathrm{ml}$ and a half-life of $3.5 \mathrm{~h}$. The treatment of ACY-1083 showed effect in preventing cisplatin-induced mechanical allodynia as well as reversing spontaneous pain and numbness induced by cisplatin in mice models. This work provided substantial evidence that inhibition of HDAC6 could serve as a promising novel therapeutic strategy for the prevention and treatment of chemotherapy-induced peripheral neuropathy [95]. ACY-738, ACY-775 and ACY-1215 were also 
investigated for the treatment of mutant B small heat shock protein B1-induced CMT2. In an animal study, even with low administration dose of ACY-738 (i.p. $3 \mathrm{mg} / \mathrm{kg}$ ), ACY-775 (i.p. $3 \mathrm{mg} / \mathrm{kg}$ ) and ACY-1215 (i.p. $30 \mathrm{mg} / \mathrm{kg}$ ), all these compounds exhibited significant effects on both the motor and sensory defects in CMT2 mice. This study demonstrated that the pharmacological inhibition of HDAC6 might be a potential therapy against CMT2 [96].

Senger $e t$ al. developed an oxazole-containing HDAC6 inhibitor 36, which inhibited HDAC6 activity with an $\mathrm{IC}_{50}$ of $59 \mathrm{nM}$ and had a selectivity index of $>200$ over HDAC1 and HDAC8 [97]. Oxazole has been extensively exploited in drug discovery and was directly connected to the ZBG in this case, which potentially created $\pi$ $\pi$ stacking interactions with Phe566 and Phe520 in HDAC6. In addition, 36 had a low micromolar-range antiproliferative activity on HL60 cells. Due to the relatively small size of 36, it has the potential to be further optimized by attaching common branched substitution patterns in the capping region. Shen and co-workers reported new HDAC6 inhibitors with an isoxazole moiety directly connected to ZBG [98]. Compound 37 (SS-208) exhibited good potency toward HDAC6 $\left(\mathrm{IC}_{50}=12 \mathrm{nM}\right)$ and excellent selective over other HDACs. Remarkably, the co-crystal structure of 37 with drHDAC6-CD2 revealed its bidentate zinc coordination, which was different from the preferred monodentate coordination for most phenylhydroxamate-based HDAC6 antagonists. Compound 37 showed weak activity on the viability of murine SM1 melanoma cells at the cellular level. However, in a murine SM1 syngeneic melanoma mouse model, it could effectively impair tumor growth through an immune-mediated antitumor mechanism.

Blackburn et al. reported the discovery of the 4-(aminomethyl)- $N$-hydroxybenzamide derivatives as potent leads with unexpected HDAC6 selectivity over the other subtypes by screening a compound library [99]. Further optimization focused on constraining the conformations by creating a fused heterocycle from the amide group yielded compound 38, which showed enhanced HDAC6 selectivity and inhibitory activity $\left(\mathrm{IC}_{50}=36 \mathrm{nM}\right)$. The unique selectivity may because HDAC6 has a wider catalytic channel compared with other HDACs, allowing for the recruitment of bulkier linker elements. A follow-up study showed that by replacing the phenyl ring by pyrrole, the binding affinity of analog 39 slightly increased [100].

Song and co-workers performed a virtual screening campaign in the surface and channel region of the HDAC6, and introduced the ZBG into the hit compounds through structure-based drug design [101]. Using this approach, compound $\mathbf{4 0}$ was discovered as a novel anthraquinone-based HDAC6-specific inhibitor. The study established a novel approach to the design of HDAC6-selective inhibitors. By introducing an adamantane cap group, Strebl et al. reported a highly brain penetrant HDAC6 inhibitor 41 (Bavarostat) with an $\mathrm{IC}_{50}$ value of $60 \mathrm{nM}$ against HDAC6, and it had more than 80-fold selectivity over other HDACs. Due to its unique feature of penetrating the blood-brain barrier (BBB), it was used to develop a positron emission tomography probe for HDAC6 imaging in the living brain. This study provides a potential probe for the first-in-human neurochemical HDAC6 imaging, and it might have promising applications in the diagnosis of HDAC6 related neurological diseases [102].

\section{Nonhydroxamate acid-derived inhibitors}

The chemical structures of other HDAC6-specific inhibitors are given in Figure 4. Although hydroxamate acid is the most frequently used ZBG for HDAC6 inhibitors, underlying concerns about their PK liabilities motivated researchers to develop alternative ZBGs. Of note, a decent HDAC6 activity/selectivity profile was achieved by adding sulfur-containing ZBGs. For instance, Kozikowski et al. reported compound 42, an HDAC6-selective inhibitor with a mercaptoacetamide ZBG [103]. Remarkably, 42 effectively protected cortical neurons from oxidative stress-induced death without apparent toxicity, whereas analogs with hydroxamate acid ZBG displayed toxicity at high concentration, highlighting the importance of isoform selectivity when targeting neurodegenerative disease. However, further in-depth studies such as BBB penetration and ADME profile for this ZBG need to be carefully evaluated. Similarly, by using the mercaptoacetamide as ZBG, Kalin et al. reported another inhibitor 43 with an $\mathrm{IC}_{50}$ value of $280 \mathrm{nM}$ against HDAC6 and high selectivity over HDAC1-5 [104]. Notably, the authors also separated the enantiomer of compound 43 and found the $S$-isomer completely abolished the binding $\left(\mathrm{IC}_{50}>30 \mu \mathrm{M}\right)$, suggesting that the chiral center is critical for maintaining HDAC6 activity. Compound $\mathbf{4 3}$ showed a fully neuroprotective effect at $10 \mu \mathrm{M}$ in the oxidative stress-induced primary cortical neuron without inducing toxicity. Thione derivatives represent another class of novel ZBG. Patil et al. reported compound 44 bearing a 3-hydroxypyridin-2-thione ZBG showed an $\mathrm{IC}_{50}$ value of $306 \mathrm{nM}$ against HDAC6 without inhibiting HDAC1 [105]. Later on, further optimization on linker and capping regions led to the discovery of lead 45, which inhibited HDAC6 and HDAC8 with IC 50 values of 356 and $2831 \mathrm{nM}$, respectively [106]. Of note, the substitution groups on the phenyl ring of $\mathbf{4 5}$ had a significant impact on the HDAC6/HDAC8 selectivity profile. Muthyala et al. reported a novel HDAC6-specific 

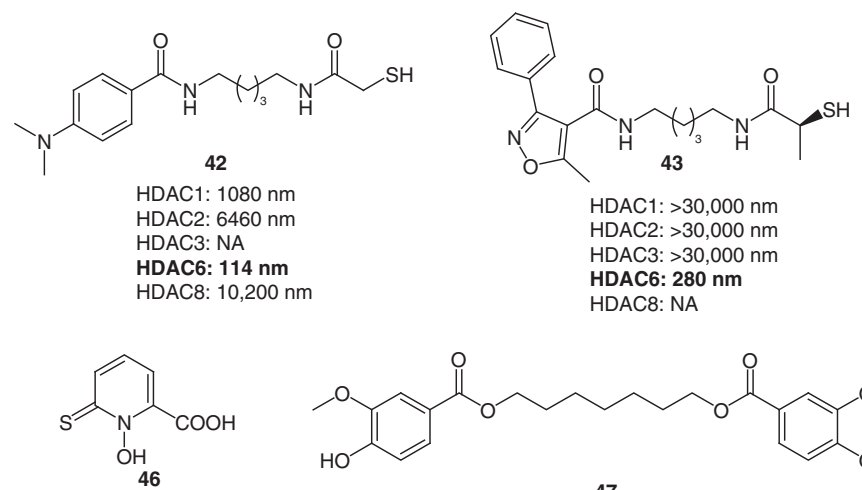

HDAC1: $43,000 \mathrm{~nm}$ HDAC2: $710,000 \mathrm{~nm}$ HDAC3: NA HDAC6: $150 \mathrm{~nm}$ HDAC8: $3300 \mathrm{~nm}$

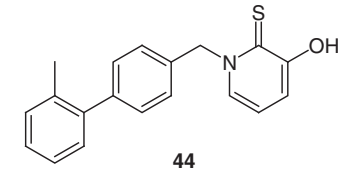

HDAC1: $>10,000 \mathrm{~nm}$ HDAC2: NA

HDAC3: NA

HDAC6: $306 \mathrm{~nm}$

HDAC8: $3105 \mathrm{~nm}$

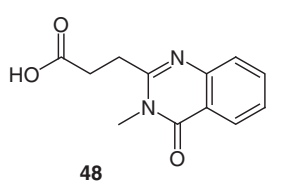

HDAC1: NA

HDAC2: NA

HDAC3: NA

HDAC6: 2300 nm (ZnF-UBD)

HDAC8: NA

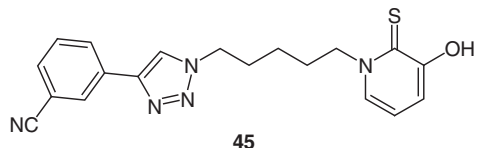

45

HDAC1: >10,000 nm

HDAC2: NA

HDAC3: NA

HDAC6: $356 \mathrm{~nm}$

HDAC8: $2831 \mathrm{~nm}$

Figure 4. The structures of nonhydroxamate acid-derived histone deacetylase 6 inhibitors.

HDAC6: Histone deacetylase 6; NA: Not active.

antagonist 46 with a unique 1-hydroxypyridine-2-thione ZBG [107]. Compound 46 showed good inhibitory activity of HDAC6 with an $\mathrm{IC}_{50}$ of $150 \mathrm{nM}$, and at least 22-fold selectivity over other tested HDACs. Seidel and co-workers discovered compound 47, a selective HDAC6 inhibitor without a typical ZBG [108]. Compound $\mathbf{4 7}$ has moderate activity against HDAC6 $\left(\mathrm{IC}_{50}=0.2 \mu \mathrm{M}\right)$ while sparing other HDAC isoforms $\left(\mathrm{IC}_{50}\right.$ range: $\left.10-100 \mu \mathrm{M}\right)$.

HDAC6 has a unique ZnF-UBD; however, pharmacologically displacing ubiquitin from this domain was largely unexplored. Notably, the potential inhibitors for this specific domain are likely to possess totally different structural features. Recently, Freitas et al. discovered several HDAC6 inhibitors targeting this domain using virtual screening [54]. After hit expansion, compound 48, a more potent and drug-like candidate, was identified with low micromolar activity. The quinazolinone scaffold of $\mathbf{4 8}$ represented a significant breakthrough in the potency and ligand efficiency in this series. Most importantly, a series of the cocrystal structures of these ligands in complex with the ZnF-UBD had successfully been solved, providing structural insight for further optimization. Moreover, crystal structure analysis demonstrated that one compound induced conformational rearrangements of the binding site, making it possible to improve the binding affinity further.

\section{Chimeric HDAC6 inhibitors}

MTD design is an active research area in medicinal chemistry. MTDs are expected to be more effective than singletarget inhibitors considering their synergistic effects between different pathways. Compared with combinationbased therapies, MTDs have several potential advantages, including less adverse drug-drug interactions, simpler PK and better patient compliance. MTDs with broad-spectrum anti-HDAC activities have been summarized in recent reviews [109-111]. Two representative MTDs, CUDC-101 (targeting EGFR/HER2/HDAC) and CUDC-907 (targeting PI3Ks/HDAC), have been shown to overcome drug resistance to single-agents and entered clinical trials. We will focus on the hybrids that selectively bind to HDAC6 in this review. As we described previously, the caps in HDAC6 inhibitors are highly diverse and tolerated to extensive structural modifications. This characteristic feature of HDAC6 inhibitors allows medicinal chemists to convert them into MTDs by introducing other pharmacophores into the capping region. The chemical structures of chimeric HDAC6 inhibitors are summarized in Figure 5.

The PI3K/AKT/mTOR pathway, a critical signal transduction system that transduces signals from cell surface receptors to downstream effectors, is one of the most commonly activated signaling pathways in human cancer [112]. The limited clinical efficacy of PI3K inhibitors promotes the development of combinational therapies or chimeric PI3K MTDs. Thakur et al. developed a series of chimeras by incorporating HDAC ZBG into the solvent-exposed region of PI3K inhibitor idelalisib [113]. The linker part was systematically explored, and compound 49 with a phenylhydroxamate motif maintained a high potency against $\mathrm{PI} 3 \mathrm{~K} \gamma$ and $\mathrm{PI} 3 \mathrm{~K} \delta$ with $\mathrm{IC}_{50}$ values of 9.0 and $7.0 \mathrm{nM}$, respectively. On the other hand, 49 displayed vigorous HDAC6 binding affinity $\left(\mathrm{IC}_{50}=12 \mathrm{nM}\right)$, which is more than 39-fold selectivity over other HDACs. In vitro, compound 49 exhibited antiproliferative activity against multiple cancer cell lines. Remarkably, 49 induced necrosis in several mutant and FLT3-resistant acute myeloid 

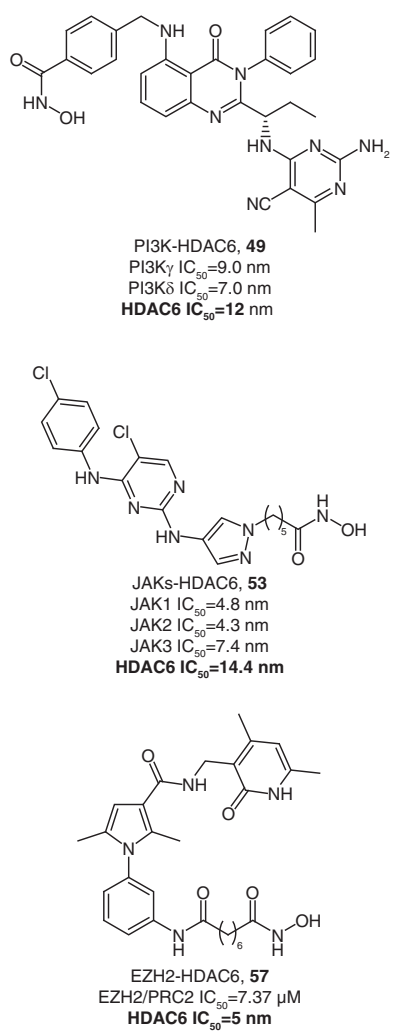
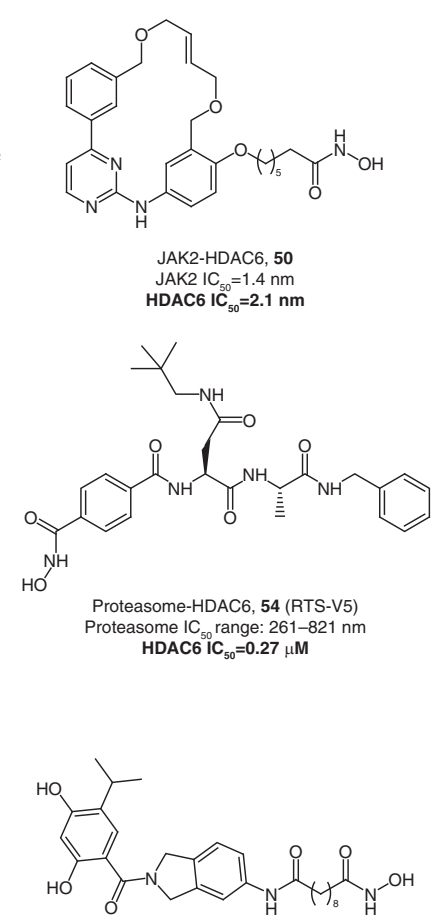

HSP90-HDAC6, 58
HSP90a IC
50 HDAC6 $\mathrm{IC}_{50}=4.3 \mathrm{~nm}$
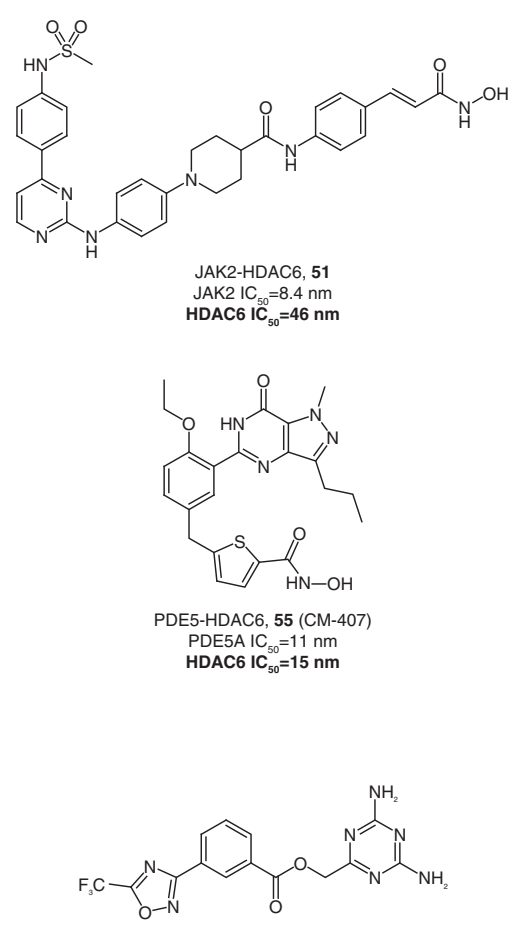

HSP90-HDAC6, 59
32.1\% HSP90 inhibition at $50 \mu \mathrm{M}$
$\mathbf{5 3} \%$ HDAC6 at $\mathbf{5} \mu \mathrm{M}$<smiles></smiles>

JAK2-HDAC6, 52 JAK2 $\mathrm{IC}_{50}=41 \mathrm{pm}$
HDAC6 IC

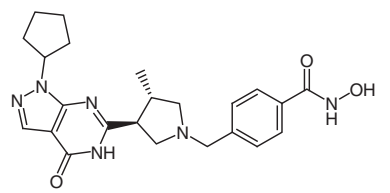

PDE9-HDAC6, 56 (CM-695) PDE9 IC
HDAC6 $I C_{50}=407 \mathrm{~nm}$<smiles>CC(COc1no[n+]([O-])c1S(=O)(=O)c1ccccc1)NO</smiles>
NO donor-HDAC6, 60

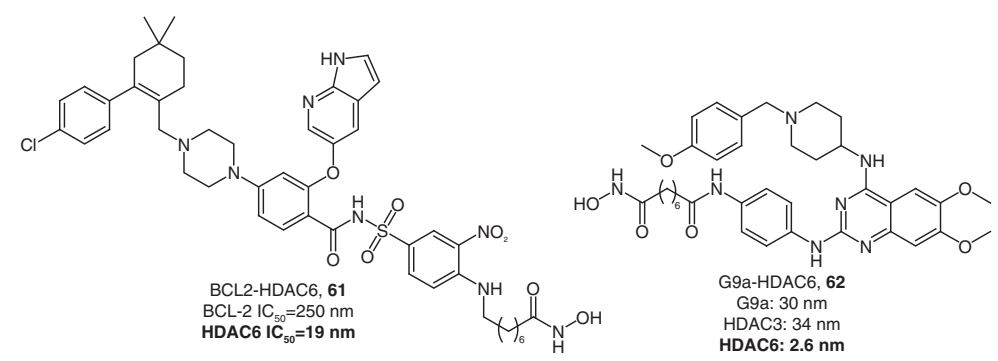

Figure 5. The chemical structures of chimeric histone deacetylase 6 inhibitors.

HDAC6: Histone deacetylase 6; HSP90: Heat shock protein 90.

leukemia (AML) cell lines as well as primary blasts from AML patients without causing considerable cytotoxicity against normal PBMCs, NIH3T3 and HEK293 cells. The favorable microsomal stability and PK profile warrants in vivo efficacy studies and further lead optimization efforts are currently underway.

JAKs are a family of intracellular tyrosine kinases that can trigger phosphorylation and dimerization of the STATs upon activation by various cytokines. Subsequent translocation of dimerized STATs into the cell nucleus can regulate transcription of selected genes [114]. The known four subtypes of JAKs are JAK1, JAK2, JAK3 and TYK2, and among which, JAK2 is relevant to tumor growth and progression. JAK2 antagonists have been evaluated in clinical trials for cancer treatment and drug resistance has been observed [115]. Therefore, several JAK2-involved MTDs have been developed based on synergistic combination therapies. Yang et al. developed a series of JAK2HDAC dual inhibitors derived from JAK2/FLT3 inhibitor pacritinib [116]. The hybridization of SAHA with pacritinib from the JAK 2 solvent channel resulted in the discovery of 50, a novel dual JAK2-HDAC inhibitor with single-digit nanomolar potency against JAK2 $\left(\mathrm{IC}_{50}=1.4 \mathrm{nM}\right)$ and HDAC6 $\left(\mathrm{IC}_{50}=2.1 \mathrm{nM}\right)$. Also, 50 displayed decent JAK2 and HDAC6 selectivity over other JAKs (>50-fold) and HDACs (>23-fold), respectively. In vitro, broad antiproliferative activities were observed across multiple solid and hematological tumor cell lines. Further research confirmed that $\mathbf{5 0}$ was able to regulate both JAK-STAT and HDAC pathways at low concentrations. This 
work provides the first isozyme-selective dual JAK2-HDAC6 inhibitors that might serve as useful tool compounds for further studies of multiple pathway inhibition.

Later on, Huang and coworkers reported a new series of dual JAK2/HDAC inhibitors derived from JAK2 inhibitor momelotinib (also known as CYT-387) [117]. Structure-based design by linking a conjugated hydroxamate group to the solvent exposure region of CYT-387 yielded JAK2/HDAC dual inhibitor $\mathbf{5 1}$ with $\mathrm{IC}_{50}$ values of 8.4 and $46 \mathrm{nM}$ toward JAK2 and HDAC6, respectively. The isoform selectivity of $\mathbf{5 1}$ over other JAKs and HDACs was confirmed using enzyme inhibition assays, and the HDAC binding profile appeared to be more sensitive to linker modifications. Compound $\mathbf{5 1}$ exhibited good cellular cytotoxicity against hematological tumor cell lines as a single-agent and significantly prolonged the survival of mice bearing human erythroleukemia (HEL) xenografts. In addition, the combination treatment of $\mathbf{5 1}$ with fluconazole extended the survival of mice infected with resistant Candida albicans. This work highlighted the therapeutic potential of JAK2/HDAC6 dual inhibitors in treating AML and invasive fungal infections.

Another work by Yao and coworkers merged ruxolitinib, a marketed JAK inhibitor, with SAHA to yield several chimeras [118]. Notably, compound $\mathbf{5 2}$ displayed an unprecedented binding affinity toward JAK2 $\left(\mathrm{IC}_{50}=41 \mathrm{pM}\right)$ and excellent HDAC6 inhibitory activity $\left(\mathrm{IC}_{50}=0.25 \mathrm{nM}\right)$. Among all designed compounds in this study, 52 showed the most potent antiproliferative activities across four tested solid tumor cell lines. By using a similar strategy, Liang et al. developed a new series of pyrimidin-2-aminopyrazol hydroxamate derivatives as dual JAKHDAC inhibitors. Different from compounds $\mathbf{5 0 - 5 2}$ that selectively targeting JAK2 and HDAC6, compound $\mathbf{5 3}$ was the most potent in this study and simultaneously antagonized JAK1-3 ( $\mathrm{IC}_{50}$ range: 4-8 nM) and HDAC6 $\left(\mathrm{IC}_{50}=14.4 \mathrm{nM}\right)$. Remarkably, 53 exhibited superior antiproliferative activity against HEL cells with an $\mathrm{IC}_{50}$ value of $9 \mathrm{nM}$, which is more potent than the combinational treatment of SAHA and ruxolitinib. In vivo, compared with the combination group $(\mathrm{TGI}=57 \%), 53(100 \mathrm{mg} / \mathrm{kg}$, i.p.) showed a moderate antitumor potency $(\mathrm{TGI}=36 \%)$ in HEL xenograft model, probably because of the short half-life $\left(T_{1 / 2}=0.26\right)$, rapid clearance and low tumor exposure. Optimization that focuses on improving the PK parameters may further increase in vivo efficacy [119].

Combination therapy using HDACi and proteasome inhibitor can simultaneously block proteasome and aggresome pathways and induce apoptosis [120]. Bhatia et al. developed the first-in-class dual HDAC-proteasome inhibitors by hybridization of noncovalent proteasome inhibitor ML-16 with a selective HDAC6 inhibitor [121]. RTS-V5 (54), a potent dual HDAC-proteasome ligand, exhibited good binding affinity toward HDAC6 $\left(\mathrm{IC}_{50}=0.27 \mu \mathrm{M}\right)$ with moderate isozyme-selectivity. Besides, RTS-V5 was able to selectively block the chymotrypsin-like proteasome activity in selected leukemic cell lines (HL-60, SEM and SUP-B15r) without interrupting trypsin- and caspase-like proteasome activities. Noteworthy, the crystal structures of RTS-V5 in complex with HDAC6 and yeast 20 S proteasome were successfully solved in this study. Specifically, the authors highlighted that the bulky phenylhydroxamate group in RTS-V5 preferably binds to HDAC6 but not HDAC1-3, which might be responsible for the low toxicity of this compound. Also, RTS-V5 showed potent anticancer activity against AML, chronic myeloid leukaemia, multiple myeloma and patient-derived B-cell precursor acute lymphoblastic leukemia cells. This work provides useful information for future structure-based optimization of dual HDAC-proteasome inhibitors.

cAMP and cGMP are intracellular second messengers that play an essential role in signal transduction by regulating many critical physiological and pathophysiological processes such as cellular growth, differentiation and proliferation. PDEs are a superfamily of conserved enzymes (PDE1 to PDE12) that control the intracellular levels of cyclic nucleotides by hydrolyzing cAMP and cGMP, thereby playing an essential role in regulating signal transduction mediated by cAMP and cGMP [122]. Emerging evidence supports that PDEs are therapeutic targets for various diseases, including but not limited to neurodegenerative disorders. For example, the inhibition of PDE5, PDE6 and PDE9 results in cGMP accumulation, which may affect neuronal signaling and ultimately promote memory-related gene transcription via the activation of CREB. Similarly, HDACs have gained attention as therapeutic targets for AD. In particular, HDAC6 inhibition has been implicated in the degradation of misfolded proteins such as phosphorylated tau (pTau) and $\mathrm{A} \beta$ Considering the multifaceted nature of $\mathrm{AD}$, MTDs targeting both PDE and HDAC are promising agents for the treatment of AD. Rabal and colleagues discovered a series of PDE5-HDAC6 dual inhibitors derived from sildenafil [123]. Compound 55 (CM-407), the most potent candidate in this series, displayed strong binding affinities toward PDE5A $\left(\mathrm{IC}_{50}=11 \mathrm{nM}\right)$ and HDAC6 $\left(\mathrm{IC}_{50}=15 \mathrm{nM}\right)$ with a superior selective profile over class I HDAC isozymes. Of note, CM-407 also potently inhibitor PDE6 with an IC 50 of $1.4 \mathrm{nM}$. In vitro, CM-407 caused downregulation of the AD-related markers hAPP and pTau in Tg2576 neurons. In vivo, CM-407 treatment led to increased phosphorylated CREB (pCREB) levels compared with the untreated 
group, suggesting CM-407 penetrated the BBB and achieved its functional response in the hippocampus. After 2 weeks of CM- 407 treatment in vivo, partial restoration of the memory impairment was observed in Tg2576 mice. Interestingly, an analog of CM-407 with moderate class I HDAC inhibitory activity showed better in vivo efficacy, raising the question of whether targeting more HDACs would be a better option. PDE9 has the highest affinity for cGMP among all PDEs and is overexpressed in AD brains. Therefore, the same research group developed a new series of dual PDE9-HDAC inhibitors with diverse isoform-selectivity profiles against HDACs, including panHDAC, HDAC6 selective and class I selective [124]. Among all designed chimeras, compound 56 (CM-695) with decent potency and selectivity against HDAC6 and PDE9 was able to upregulate levels of Ac-tubulin and pCREB at the cellular level. In addition, CM-695 possessed acceptable in vitro ADME profiling, although cardiovascular alerts of this compound should be carefully taken into account $\left(\mathrm{hERG} \mathrm{IC}_{50}=1.43 \mu \mathrm{M}\right)$. In the aged $\mathrm{Tg} 2576$ mice model, chronic treatment of dual PDE9-HDAC6 inhibitor CM-695 showed a positive effect in reversing the AD phenotype [125].

EZH2 is a HMT that catalyzes the addition of methyl groups to H3K27 [126]. Overexpression or mutation of EZH2 has been observed in multiple cancers, and inhibition of EZH2 blocks the histone methylation of tumor suppressor genes. HDACs and EZH2 both control epigenetic maintenance of genes. Recent studies demonstrated that synergistic effects between EZH2 inhibitor and HDACi in various cancer cell lines [127]. Compound 57, a first-in-class dual EZH2-HDAC inhibitor, showed a superior inhibitory activity against HDAC6 $\left(\mathrm{IC}_{50}=5 \mathrm{nM}\right)$, which is at least 22-fold more potent than other tested HDAC isozymes [128]. Compound 57 caused the upregulation of acetyl- $\alpha$-tubulin and downregulation of $\mathrm{H} 3 \mathrm{~K} 27 \mathrm{me} 3$, G1 cell cycle arrest, and antiproliferative effects in leukemia U937 and rhabdomyosarcoma RH4 cells. Moreover, $\mathbf{5 7}$ hampered epithelial to mesenchymal transition in glioblastoma U87 cells.

Hsp90 is a chaperone protein that is responsible for protein folding and stabilization [129]. Hsp90 inhibitors are investigated in the clinic as anticancer agents. On the other hand, HDAC6 is capable of deacetylating Hsp90 and modulating its chaperone activity. Ojha et al. reported isoindoline-based dual HDAC6-Hsp90 inhibitors based on the synergistic reports of HDAC6 inhibitors with Hsp90 inhibitors. Compound $\mathbf{5 8}$ displayed potent inhibitory activity toward both HDAC6 $\left(\mathrm{IC}_{50}=4.3 \mathrm{nM}\right)$ and $\mathrm{Hsp} 90\left(\mathrm{IC}_{50}=46.8 \mathrm{nM}\right)$. In vitro, 58 modulated the levels of signatory biomarkers associated with HDAC6 and HSP90 inhibition as well. Besides, $\mathbf{5 8}$ exhibited growth inhibitory effects on $\mathrm{A} 549$ (with $\mathrm{GI}_{50}=0.76 \mu \mathrm{M}$ ) and $\mathrm{H} 1975\left(\mathrm{GI}_{50}=0.52 \mu \mathrm{M}\right)$ cell lines. In human H1975 xenografts model, 58 suppressed tumor growth both in monotherapy (TGI $=69.7 \%$ ) and combination therapy with afatinib (TGI $=72.3 \%$ ). Further, 58 could reduce IFN- $\gamma$ induced PD-L1 expression in H1975 cells, indicating its capability of modulating the immunosuppressive environment of tumor area [130]. Using an integrated computational strategy, Pinzi and co-workers developed a new series of dual HDAC6-Hsp90 inhibitors [131]. Ten selected candidates were evaluated in bioassays, and compound $\mathbf{5 9}$ was identified as a dual inhibitor of HDAC6 and Hsp90. However, 59 exhibited weak inhibition against HDAC6 (53\% inhibition at $5 \mu \mathrm{M})$ and $\mathrm{Hsp} 90(32.1 \%$ inhibition at $50 \mu \mathrm{M})$. Nevertheless, this study provides a novel scaffold of dual Hsp90/HDAC6 inhibitors that may serve as a valuable starting point for further optimization.

Nitric oxide is a chemical species that is best known as a vasodilator. Accumulating evidence reveals that it is also associated with multiple cancer-related events such as tumor proliferation, metastasis, angiogenesis and apoptosis [132]. By attaching a nitric oxide donor to the capping region of HDACi, Duan and colleagues designed a novel series of phenylsulfonylfuroxan-based hydroxamates as potential dual-function molecules [133]. Compound $\mathbf{6 0}$ could simultaneously promote the nitric oxide release and inhibit HDAC6 activity ( $\mathrm{IC}_{50}=7.4 \mathrm{nM}$ ). In addition, $\mathbf{6 0}$ exhibited better antiproliferative activity against HEL cells than the parent compound SAHA. Furthermore, 60 was orally bioavailable and displayed good antitumor activity in the HEL xenograft-bearing mice (TGI $=48 \%$ ).

$\mathrm{Bcl}-2$ family proteins are critical regulators of the intrinsic apoptotic pathway [134]. Three anti-apoptotic members, BCL-2, BCL-X $\mathrm{X}_{\mathrm{L}}$ and MCL-1, are validated anticancer targets. Zhou et al. designed several of BCL-2-HDAC dualtarget inhibitors derived from venetoclax (ABT-199) [135]. Compound 61 showed binding affinity toward HDAC6 and BCL-2 with IC $_{50}$ values of 19 and $250 \mathrm{nM}$, respectively. Moreover, it exhibited good cytotoxicity against human multiple myeloma cell line RPMI-8226.

Epigenetic modification of acetylation and methylation have been proven to play a critical role in complex malarial parasite life cycles. Recently, by merging the pharmacophore of an HDACi and G9a (also known as EHMT2, a histone methyltransferase enzyme) inhibitor, Soumyanarayanan et al. reported a series of HDAC-G9a dual inhibitors, among which, compound 62 exhibited low nanomolar potency against HDAC6 $\left(\mathrm{IC}_{50}=2.6 \mathrm{nM}\right)$ and HDAC3 $\left(\mathrm{IC}_{50}=34 \mathrm{nM}\right)$ with at least 300 fold selectivity against other HDACs. At the same time, $\mathbf{6 2}$ showed 
G9a inhibitory activity with an $\mathrm{IC}_{50}$ value of $30 \mathrm{nM}$. Remarkably, in vitro study demonstrated that $\mathbf{6 2}$ showed antimalarial effects, especially for chloroquine-resistant strain $\mathrm{K} 1$ of P.falciparum. This study provided a promising potential application of novel HDAC-G9a dual inhibitors to treat malaria [136].

\section{HDAC6 PROTACs}

PROTACs are heterobifunctional molecules that can recruit the protein of interest (POI) to the E3 ubiquitin ligase and trigger subsequent ubiquitination and proteasomal degradation of POI. The discovery of small-molecule E3 ligase ligands promoted the rapid development of PROTAC technology that has arisen as a revolutionary approach in pharmaceutical discovery. Tremendous efforts have been made in this area and efficient degradation of diverse POIs, including nuclear receptors, protein kinases, epigenetic regulators, anti-apoptotic proteins and transcription factors, has been achieved $[36,37,137]$. So far, celeblon (CRBN) and von Hippel-Lindau (VHL) are the most explored E3 ligases in this area. Two PROTACs developed by Arvinas Inc. (CT, USA) are currently under clinical trials for the treatment of prostate cancer and breast cancer, respectively.

Despite the expenditure of vast effort on the development of HDACis, clinical success for the treatment of solid tumors using HDACi as a single-agent has yet to be achieved, which is probably because of the insufficient concentrations in tumor tissue. Moreover, dose-limiting adverse effects such as hERG-related cardiotoxicity also limit the clinical performance [138,139]. Therefore, novel strategies aiming to improve drug efficacy and reduce toxicity may lead to new drug candidates with superior drug properties.

Thanks to the unique mechanism of action, PROTAC degraders possess several advantages over conventional inhibitors, such as improved potency, prolonged suppression of POI and potential tissue/cell type selectivity [140,141]. By converting an HDACi into a PROTAC, several desirable properties might be expected. First, since PROTACs act via a catalytic mechanism of action, even when the tissue concentration is relatively low, it is still possible to achieve in vivo therapeutic effect. Second, since protein recovery usually takes time after being depleted, HDAC degraders are likely to have a longer duration of action in comparison to traditional HDACi. Further, on account of the involvement of POI-PROTAC-E3 ternary complexes, specific degradation may be achieved even by recruiting a promiscuous ligand as the warhead. The increased specificity might alleviate the adverse effects related to unbiased inhibition of multiple isoforms, which is especially crucial for diseases other than cancer.

Recently, several studies using the PROTAC approach for HDAC degradation were reported [25,39-42,142-144]. The majority of those PROTACs are HDAC6-selective, whereas the others are targeting class I HDAC(s) for degradation (Figure 6). Using a strategy that can quickly build up the HDACi library, Yang et al. discovered the first HDAC6 degrader in 2018 [25]. Four PROTACs recruiting CRBN E3 ligase were designed and synthesized. Among them, 63 (dHDAC6) selectively degrades HDAC6 while sparing other isoforms, including HDACs 1, 2 and 4. Moreover, the upregulation of tubulin acetylation was observed, confirming the effect of HDAC6 suppression. Further study revealed that the rapid degradation of HDAC6 along with the upregulation of acetylated $\alpha$-tubulin levels started around $2 \mathrm{~h}$ after treating MCF-7 breast cancer cells with $2 \mu \mathrm{M}$ of 63 . MM.1S multiple myeloma cells appeared to be more sensitive to degrader $63\left(\mathrm{DC}_{50}=9.1 \mathrm{nM}\right)$ [40] compared with MCF7 cells $\left(\mathrm{DC}_{50}=34 \mathrm{nM}\right)$. Of note, the warhead binds to several HDAC isozymes, and the specific degradation of HDAC6 is unexpected. Similar examples of additional selectivity for PROTACs over inhibitors were previously reported [145-147]. It is also worth noting that the hydrazone-containing linker may not be suitable for further studies due to poor hydrolytic stability.

An and coworkers developed a new class of PROTACs targeting HDAC6 by conjugating selective HDAC6 inhibitor NextA and CRBN ligand pomalidomide [39]. PROTAC 64 (NP8) stood out among its analogs as the best degrader of HDAC6. Treatment of HeLa cells with $100 \mathrm{nM}$ NP8 for 24 hours resulted in remarkable degradation of HDAC6, whereas the protein levels of HDACs 1, 2 and 4 were not affected. MM.1S cell line displayed the best sensitivity to NP8 treatment $\left(\mathrm{DC}_{50}=3.8 \mathrm{nM}\right)$. Furthermore, NP8 exhibited slightly improved antiproliferative activity in the MM.1S cell line $\left(\mathrm{GI}_{50}=1.21 \mu \mathrm{M}\right)$ compared with the parent compound NextA $\left(\mathrm{GI}_{50}=2.25 \mu \mathrm{M}\right)$. In this study, the direct visualization of HDAC6 level change was achieved by fusing an enhanced green fluorescence protein to HDAC6.

CRBN ligands, including pomalidomide and lenalidomide, are immunomodulatory drugs that can recruit IKZFs for induced proteasomal degradation, which is believed to be responsible for their antiproliferative activity in multiple myeloma [148]. While the CRBN-mediated IKZF degradation is considered to be the off-target effect for most PROTACs, Wu et al. proposed that HDAC6-IKZFs dual degraders would have an enhanced antimyeloma activity based on the synergistic effect of HDAC6 inhibitors and immunomodulatory drugs in myeloma 

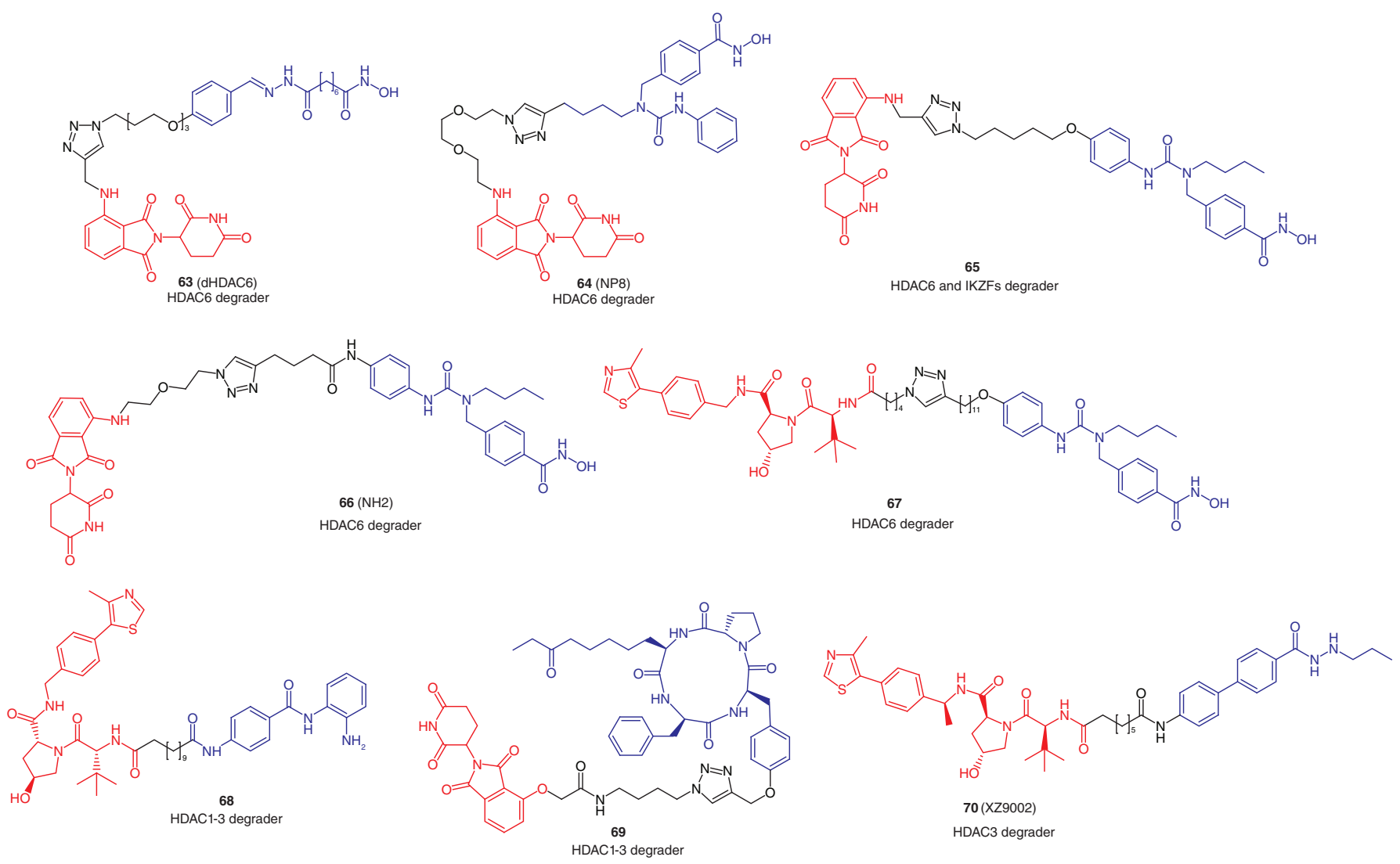

Figure 6. Reported proteolysis targeting chimeras targeting histone deacetylase(s). Histone deacetylase binding warheads are shown in blue, E3 binding motifs are shown in red and linkers are shown in black. HDAC6: Histone deacetylase 6; HSP90: Heat shock protein 90.

therapy [149]. Therefore, several NextA-derived PROTACs recruiting CRBN E3 ligase were developed, and their HDAC degradation efficacy was evaluated in MM.1S cell line [40]. Compared with the first HDAC6 degrader, 63, several new degraders can induce a more substantial degradation effect. Surprisingly, only PROTAC $\mathbf{6 5}$ but not other analogs can simultaneously deplete HDAC6 $\left(\mathrm{DC}_{50}=1.6 \mathrm{nM}\right)$ and IKZFs. Importantly, the protein level of HDAC6 was not fully recovered within $48 \mathrm{~h}$ of drug washout, suggesting a long duration of HDAC6 suppression. The viability study of degrader $\mathbf{6 5}$ in MM.1S cells revealed the advantage of dual-targeting HDAC6 and IKZFs over either NextA, pomalidomide, or their combination.

Yang et al. also introduced the linker to the benzene ring of NextA and developed a potent HDAC6-selective degrader 66 (NH2) with a $\mathrm{DC}_{50}$ of $3.2 \mathrm{nM}$ in MM.1S cells [41]. Co-incubation of Mino, Jeko-1, HUVEC and MDA-MB-231 cells with $100 \mathrm{nM} \mathrm{NH} 2$ led to significant degradation of HDAC6, suggesting NH2 may chemically knockdown cellular HDAC6 in various cell lines. Molecular docking simulation on the HDAC6-PROTAC-CRBN ternary complexes revealed that NH2 (66) and NP8 induced different protein-protein interaction interfaces to exert the similar degradation profile.

Compared with CRBN-based PROTACs, VHL-based degraders do not have reported neo-substrates such as IKZFs that can lead to potential off-target effects. Tang lab developed the first VHL-based PROTACs targeting HDAC6 for proteasomal degradation [42]. The $\mathrm{DC}_{50}$ of the most potent degrader in this series, PROTAC 67, is $7.1 \mathrm{nM}$ in MM.1S cells. Besides, the selective increase of acetylation against tubulin over histone was observed while the IKZF levels were not affected, indicating a high selectivity of $\mathbf{6 7}$. Thus, PROTAC $\mathbf{6 7}$ might serve as a specific chemical probe to explore HDAC6-related biological functions.

Overall, HDAC6 appears to be sensitive to PROTAC-mediated degradation. That is probably due to the predominant cytoplasmic localization of HDAC6, whereas most other HDACs are localized in the nucleus [150]. The first example of PROTAC-induced HDAC depletion other than HDAC6 was achieved by recruiting class I selective HDACi CI-994 as the warhead [142]. PROTAC 68, the most active degrader in this series, was able to 
downregulate HDACs 1-3 and upregulate histone acetylation levels. By conjugating a macrocyclic tetrapeptide HDACi with CRBN ligand, compound 69 is capable of degrading HDACs 1-3 in HEK293T cells [143]. Both PROTACs 68 and $\mathbf{6 9}$ are nonselective class I HDAC degraders, and the effect against HDAC3 degradation was relatively weak. More recently, Xiao et al. reported the discovery of XZ9002 (70) as a first-in-class HDAC3-specific degrader [144]. XZ9002 efficiently suppresses clonogenic growth and has potent antiproliferative activity against multiple cancer cell lines. Furthermore, XZ9002 may serve as a useful toolkit to investigate HDAC3 biology due to its high selectivity of HDAC3 degradation.

\section{Conclusion}

HDAC6 is a unique isozyme of HDACs that catalyzes primarily cytoplasmic nonhistone proteins, therefore being recognized as a key regulator of cytoskeletal dynamics, cell migration, as well as cell-cell interactions. Besides its close relation with cancers, emerging evidence also implicates its role in neurodegeneration diseases and immune responses. Isoform-selective antagonists, chimeric inhibitors and PROTACs represent three different approaches to target HDAC6.

\section{Future perspective}

Three HDAC6-selective inhibitors, ACY-241, ACY-1215 and KA2507, are under clinical evaluation either in monotherapy or combination therapy for the treatment of solid tumors and hematological malignancies. Of note, ACY-1215 is under clinical evaluation to treat patients with diabetic neuropathic pain (NCT03176472). In addition, another two HDAC6-specific antagonists, CKD-504 and CKD-506, have entered clinical trials for the treatment of Huntington disease and arthritis. So far, the majority of HDAC6-specific inhibitors employ hydroxamic acid moiety as ZBG, which is probably because of its superior zinc-binding ability. However, from a medicinal chemistry perspective, hydroxamic acid may not be an ideal pharmacophore. First, the superior but nonselective zinc-binding affinity may cause undesirable side effects derived from other zinc-dependent enzymes, such as aminopeptidases, matrix metalloproteinases and carbonic anhydrase. Second, unfavorable PK parameters (e.g., rapid clearance in vivo and low oral bioavailability) might be associated with ineffective tissue distribution and concentration, especially in solid tumors. Moreover, it can be challenging for hydroxamic acid derivatives to penetrate the BBB due to their hydrophobic nature, limiting the applications in CNS disorders such as AD. Additionally, the hydroxamate ZBG may possess mutagenic potential due to the formation of isocyanates through Lossen rearrangement. Sulfur-containing ZBG might be an alternative option, but the physicochemical and pharmaceutical properties need to be taken into consideration. Therefore, the elaboration of the novel ZBG with desirable properties is highly valuable in the future. As the only HDAC with a ZnF-UBD, HDAC6 provides a unique way to antagonize HDAC6-mediated aggresome formation and lysosomal degradation that might have unique therapeutic potential. To date, inhibitors binding to this region all showed micromolar activity, which deserves further optimization in the future. Similarly, CD1 of HDAC6 has remained relatively less explored and requires further investigation.

Considering the limited efficacy for monotherapy in the clinic, MTD design has emerged as a novel strategy to enhance the potency and overcome the drug resistance. However, in-depth investigations on the rationally designed combination and fine-tuned activity toward different targets should be conceived carefully. Moreover, PROTACs have boomed as a revolutionary technique in drug discovery. Due to their unique mechanism of action involving the formation of ternary complexes, specific proteasomal degradation of protein of interest (here, HDAC6) can be achieved. HDAC6 appeared to be sensitive to PROTAC-mediated degradation, even using a promiscuous warhead, which is due to its cytoplasmic localization. Different from conventional inhibitors, the degradation of POI may simultaneously abolish enzymatic and nonenzymatic functions, which might lead to a more potent therapeutic effect. Nevertheless, all reported HDAC6 PROTACs are using triazole-containing linkers, and their therapeutic benefit remains to be documented in vivo. Further optimization for PROTACs should focus on linkerology as well as PK, safety and drug-like properties.

Tremendous efforts have been devoted to the development of novel small molecular modulators targeting HDAC6. With five drug candidates being evaluated in the clinic, it is worthy of further exploiting the therapeutic potential of different types of HDAC6 modulators. 


\section{Executive summary}

- Histone deacetylase 6 (HDAC6) is involved in many diseases, including cancer, neurodegenerative disease and immunology.

- Selectively targeting HDAC6 might minimize the adverse effects derived from broad inhibition of HDACs.

- HDAC6-specific inhibitors can achieve optimal binding affinity and isozyme selectivity.

- HDAC6-selective inhibitors ACY-241, ACY-1215, KA2507, CKD-504 and CKD-506 are currently under clinical trials to treat cancer, diabetic neuropathic pain or Huntington's disease.

- Compared with CD2 of HDAC6, ZnF-UBD and CD1 are less explored and requires more investigation.

- Chimeric HDAC6 inhibitors represent novel agents to improve efficacy and overcome drug resistance.

- Proteolysis targeting chimeras targeting HDAC6 can lead to significant degradation of HDAC6 and hold the potential to serve as novel HDAC6 modulators with further optimization.

- It is worthy of further exploiting the therapeutic potential of different types of HDAC6 modulators.

\section{Author contributions}

$Y$ Xiao and $X$ Zhang wrote the first draft of the manuscript and $X$ Zhang. revised the manuscript. All authors contributed to manuscript revision and approved the final version.

\section{Acknowledgements}

The authors would like to thank Dr Guangrong Zheng for thoughtful discussion and editorial assistance.

\section{Financial \& competing interests disclosure}

This work was supported in part by UFHCC MOO Research Grant P0157383. Y Xiao and X Zhang are inventors of a provisional patent application for the use of HDAC PROTACs as anticancer agents. The authors have no other relevant affiliations or financial involvement with any organization or entity with a financial interest in or financial conflict with the subject matter or materials discussed in the manuscript apart from those disclosed.

No writing assistance was utilized in the production of this manuscript.

\section{Open access}

This work is licensed under the Attribution-NonCommercial-NoDerivatives 4.0 Unported License. To view a copy of this license, visit http://creativecommons.org/licenses/by-nc-nd/4.0/

\section{References}

Papers of special note have been highlighted as: • of interest; $\bullet \bullet$ of considerable interest

1 Egger G, Liang G, Aparicio A, Jones PA. Epigenetics in human disease and prospects for epigenetic therapy. Nature 429(6990), 457-463 (2004).

2 Hubbert C, Guardiola A, Shao R et al. HDAC6 is a microtubule-associated deacetylase. Nature 417(6887), 455-458 (2002).

3 Vaijayanthi T, Pandian GN, Sugiyama H. Chemical control system of epigenetics. Chem. Rec. 18(12), 1833-1853 (2018).

4 Yoshida M, Kudo N, Kosono S, Ito A. Chemical and structural biology of protein lysine deacetylases. Proc. Jpn Acad. Ser. B Phys. Biol. Sci. 93(5), 297-321 (2017).

5 Zhang X, Yuan Z, Zhang Y et al. HDAC6 modulates cell motility by altering the acetylation level of cortactin. Mol. Cell 27(2), 197-213 (2007).

6 Deskin B, Shan B, Lasky J. Targeting the Hdac6/hsp90 axis to destabilize the notch1 receptor in non-small cell lung cancer. Am. J. Respir. Crit. Care Med. 193, A2374 (2016).

7 Kramer OH, Mahboobi S, Sellmer A. Drugging the HDAC6-HSP90 interplay in malignant cells. Trends Pharmacol. Sci. 35(10), 501-509 (2014).

8 Cohen TJ, Guo JL, Hurtado DE et al. The acetylation of tau inhibits its function and promotes pathological tau aggregation. Nat. Commun. 2, 252 (2011).

9 Parmigiani RB, Xu WS, Venta-Perez G et al. HDAC6 is a specific deacetylase of peroxiredoxins and is involved in redox regulation. Proc. Natl Acad. Sci. USA 105(28), 9633-9638 (2008).

10 Matsuyama A, Shimazu T, Sumida Y et al. In vivo destabilization of dynamic microtubules by HDAC6-mediated deacetylation. EMBO J. 21(24), 6820-6831 (2002).

11 Dallavalle S, Pisano C, Zunino F. Development and therapeutic impact of HDAC6-selective inhibitors. Biochem. Pharmacol. 84(6), 756-765 (2012). 
12 Batchu SN, Brijmohan AS, Advani A. The therapeutic hope for HDAC6 inhibitors in malignancy and chronic disease. Clin. Sci. (Lond) 130(12), 987-1003 (2016).

- Demonstrates the therapeutic hope for Histone deacetylase 6 (HDAC6) inhibitors.

13 Boyault C, Sadoul K, Pabion M, Khochbin S. HDAC6, at the crossroads between cytoskeleton and cell signaling by acetylation and ubiquitination. Oncogene 26(37), 5468-5476 (2007).

14 Kawaguchi Y, Kovacs JJ, Mclaurin A, Vance JM, Ito A, Yao TP. The deacetylase HDAC6 regulates aggresome formation and cell viability in response to misfolded protein stress. Cell 115(6), 727-738 (2003).

15 Olzmann JA, Li L, Chin LS. Aggresome formation and neurodegenerative diseases: therapeutic implications. Curr. Med. Chem. 15(1), 47-60 (2008).

16 Bobrowska A, Paganetti P, Matthias P, Bates GP. Hdac6 knock-out increases tubulin acetylation but does not modify disease progression in the R6/2 mouse model of Huntington's disease. PloS One 6(6), e20696 (2011).

17 Subramanian S, Bates SE, Wright JJ, Espinoza-Delgado I, Piekarz RL. Clinical toxicities of histone deacetylase inhibitors. Pharmaceuticals (Basel) 3(9), 2751-2767 (2010).

18 Richardson PG, Harvey RD, Laubach JP, Moreau P, Lonial S, San-Miguel JF. Panobinostat for the treatment of relapsed or relapsed/refractory multiple myeloma: pharmacology and clinical outcomes. Expert Rev. Clin. Pharmacol. 9(1), 35-48 (2016).

19 Smith N, Nucera C. Personalized therapy in patients with anaplastic thyroid cancer: targeting genetic and epigenetic alterations. J. Clin. Endocrinol. Metab. 100(1), 35-42 (2015).

20 Rivers ZT, Oostra DR, Westholder JS, Vercellotti GM. Romidepsin-associated cardiac toxicity and ECG changes: a case report and review of the literature. J. Oncol. Pharm. Pract. 24(1), 56-62 (2018).

21 Seidel C, Schnekenburger M, Dicato M, Diederich M. Histone deacetylase 6 in health and disease. Epigenomics 7(1), 103-118 (2015).

22 Dallavalle S, Pisano C, Zunino F. Development and therapeutic impact of HDAC6-selective inhibitors. Biochem. Pharmacol. 84(6), 756-765 (2012).

23 Prior R, Van Helleputte L, Klingl YE, Van Den Bosch L. HDAC6 as a potential therapeutic target for peripheral nerve disorders. Expert Opin. Ther. Targets 22(12), 993-1007 (2018).

24 Ganai SA. Small-molecule modulation of HDAC6 activity: the propitious therapeutic strategy to vanquish neurodegenerative disorders. Curr. Med. Chem. 24(37), 4104-4120 (2017).

25 Ke B, Chen YX, Tu W, Ye T, Fang XD, Yang LP. Inhibition of HDAC6 activity in kidney diseases: a new perspective. Mol. Med. 24(1), 33 (2018).

26 Kalin JH, Butler KV, Akimova T, Hancock WW, Kozikowski AP. Second-generation histone deacetylase 6 inhibitors enhance the immunosuppressive effects of Foxp3+ T-regulatory cells. J. Med. Chem. 55(2), 639-651 (2012).

27 De Zoeten EF, Wang LQ, Butler K et al. Histone deacetylase 6 and heat shock protein 90 control the functions of Foxp3(+) T-regulatory cells. Mol. Cell. Biol. 31(10), 2066-2078 (2011).

28 Aldana-Masangkay GI, Sakamoto KM. The role of HDAC6 in cancer. J. Biomed. Biotechnol. 2011, 875824 (2011).

29 Shen S, Kozikowski AP. A patent review of histone deacetylase 6 inhibitors in neurodegenerative diseases (2014-2019). Expert Opin. Ther. Target 30(2), 121-136 (2020).

-. Summarizes the latest HDAC6 inhibitors in patents from 2014-2019 and discussing pharmacological applications of selective HDAC6 inhibitors in neurodegenerative diseases.

30 Wang XX, Wan RZ, Liu ZP. Recent advances in the discovery of potent and selective HDAC6 inhibitors. Eur. J. Med. Chem. 143, 1406-1418 (2018).

31 Choi H, Kim HJ, Yang J et al. Acetylation changes tau interactome to degrade tau in Alzheimer's disease animal and organoid models. Aging Cell 19(1), e13081 (2020).

32 Fan SJ, Huang FI, Liou JP, Yang CR. The novel histone de acetylase 6 inhibitor, MPT0G211, ameliorates tau phosphorylation and cognitive deficits in an Alzheimer's disease model. Cell Death Dis. 9(6), 655 (2018).

33 Selenica ML, Benner L, Housley SB et al. Histone deacetylase 6 inhibition improves memory and reduces total tau levels in a mouse model of tau deposition. Alzheimers Res. Ther. 6(1), 12 (2014).

34 Zhang L, Liu C, Wu J et al. Tubastatin A/ACY-1215 improves cognition in Alzheimer's disease transgenic mice. J. Alzheimers Dis. 41(4), 1193-1205 (2014).

35 Hesham HM, Lasheen DS, Abouzid KA. Chimeric HDAC inhibitors: comprehensive review on the HDAC-based strategies developed to combat cancer. Med. Res. Rev. 38(6), 2058-2109 (2018).

36 Paiva SL, Crews CM. Targeted protein degradation: elements of PROTAC design. Curr. Opin. Chem. Biol. 50, 111-119 (2019).

- Highlights the recent advances in targeted protein degradation and describing the challenges that need to be addressed in order to efficiently develop potent proteolysis targeting chimeras (PROTACs).

37 Khan S, He Y, Zhang X et al. PROteolysis TArgeting Chimeras (PROTACs) as emerging anticancer therapeutics. Oncogene 39(26), 4909-4924 (2020). 
38 Yang K, Song Y, Xie H et al. Development of the first small molecule histone deacetylase 6 (HDAC6) degraders. Bioorg. Med. Chem. Lett. 28(14), 2493-2497 (2018).

39 An Z, Lv W, Su S, Wu W, Rao Y. Developing potent PROTACs tools for selective degradation of HDAC6 protein. Protein Cell 10(8), 606-609 (2019).

40 Wu H, Yang K, Zhang Z et al. Development of multifunctional histone deacetylase 6 degraders with potent antimyeloma activity. J. Med. Chem. 62(15), 7042-7057 (2019).

41 Yang H, Lv W, He M et al. Plasticity in designing PROTACs for selective and potent degradation of HDAC6. Chem. Commun. 55(98), 14848-14851 (2019).

42 Yang K, Wu H, Zhang Z et al. Development of selective histone deacetylase 6 (HDAC6) degraders recruiting Von Hippel-Lindau (VHL) E3 ubiquitin ligase. ACS Med. Chem. Lett. 11(4), 575-581 (2020).

43 Zhang Y, Gilquin B, Khochbin S, Matthias P. Two catalytic domains are required for protein deacetylation. J. Biol. Chem. 281(5), 2401-2404 (2006).

44 Zou H, Wu YQ, Navre M, Sang BC. Characterization of the two catalytic domains in histone deacetylase 6. Biochem. Biophys. Res. Commun. 341(1), 45-50 (2006).

45 Hai Y, Christianson DW. Histone deacetylase 6 structure and molecular basis of catalysis and inhibition. Nat. Chem. Biol. 12(9), 741-747 (2016).

- Provides an in-depth molecular basis of catalysis and inhibition of HDAC6.

46 Osko JD, Christianson DW. Structural basis of catalysis and inhibition of HDAC6 CD1, the enigmatic catalytic domain of histone deacetylase 6. Biochemistry 58(49), 4912-4924 (2019).

47 Porter NJ, Mahendran A, Breslow R, Christianson DW. Unusual zinc-binding mode of HDAC6-selective hydroxamate inhibitors. Proc. Natl Acad. Sci. USA 114(51), 13459-13464 (2017).

48 Osko JD, Christianson DW. Structural determinants of affinity and selectivity in the binding of inhibitors to histone deacetylase 6. Bioorg. Med. Chem. Lett. 30(8), 127023 (2020).

49 Kazantsev AG, Thompson LM. Therapeutic application of histone deacetylase inhibitors for central nervous system disorders. Nat. Rev. Drug Discov. 7(10), 854-868 (2008).

50 Shen S, Kozikowski AP. Why hydroxamates may not be the best histone deacetylase inhibitors - What some may have forgotten or would rather forget? ChemMedChem 11(1), 15-21 (2016).

51 Porter NJ, Wagner FF, Christianson DW. Entropy as a driver of selectivity for inhibitor binding to histone deacetylase 6. Biochemistry 57(26), 3916-3924 (2018).

52 Hai Y, Christianson DW. Histone deacetylase 6 structure and molecular basis of catalysis and inhibition. Nat. Chem. Biol. 12(9), 741-747 (2016).

53 Miyake Y, Keusch JJ, Wang LL et al. Structural insights into HDAC6 tubulin deacetylation and its selective inhibition. Nat. Chem. Biol. 12(9), 748-754 (2016).

-• The structure of HDAC6 CD1-CD2 in complex with NextA and homology modeling of the human CD2 domain help to understand selective inhibition of HDAC6.

54 De Freitas RF, Harding RJ, Franzoni I et al. Identification and structure-activity relationship of HDAC6 zinc-finger ubiquitin binding domain inhibitors. J. Med. Chem. 61(10), 4517-4527 (2018).

55 Harding RJ, De Freitas RF, Collins P et al. Small molecule antagonists of the interaction between the histone deacetylase 6 zinc-finger domain and ubiquitin. J. Med. Chem. 60(21), 9090-9096 (2017).

56 Wang X-X, Wan R-Z, Liu Z-P. Recent advances in the discovery of potent and selective HDAC6 inhibitors. Eur. J. Med. Chem. 143, 1406-1418 (2018).

57 Haggarty SJ, Koeller KM, Wong JC, Grozinger CM, Schreiber SL. Domain-selective small-molecule inhibitor of histone deacetylase 6 (HDAC6)-mediated tubulin deacetylation. Proc. Natl Acad. Sci. USA 100(8), 4389-4394 (2003).

58 Butler KV, Kalin J, Brochier C, Vistoli G, Langley B, Kozikowski AP. Rational design and simple chemistry yield a superior, neuroprotective HDAC6 inhibitor, tubastatin A. J. Am. Chem. Soc. 132(31), 10842-10846 (2010).

59 Ding H, Dolan PJ, Johnson GV. Histone deacetylase 6 interacts with the microtubule-associated protein tau. J. Neurochem. 106(5), 2119-2130 (2008).

60 Rodriguez-Gonzalez A, Simms-Waldrip T, Ikeda AK et al. Tubacin, an inhibitor of HDAC6, induces apoptosis of acute lymphoblastic leukemia cells in vitro and in vivo through a $\mathrm{Na}+/ \mathrm{K}+$ ATPase-dependent pathway. Blood 112(11), 671-672 (2008).

61 Hideshima T, Qi J, Paranal RM et al. Discovery of selective small-molecule HDAC6 inhibitor for overcoming proteasome inhibitor resistance in multiple myeloma. Proc. Natl Acad. Sci. USA 113(46), 13162-13167 (2016).

62 Santo L, Hideshima T, Kung AL et al. Preclinical activity, pharmacodynamic, and pharmacokinetic properties of a selective HDAC6 inhibitor, ACY-1215, in combination with bortezomib in multiple myeloma. Blood 119(11), 2579-2589 (2012).

- Provides the rationale for the preclinical case study of ACY-1215. 
63 Huang P, Almeciga-Pinto I, Jarpe M et al. Selective HDAC inhibition by ACY-241 enhances the activity of paclitaxel in solid tumor models. Oncotarget 8(2), 2694-2707 (2017).

64 Kozikowski AP, Tapadar S, Luchini DN, Kim KH, Billadeau DD. Use of the nitrile oxide cycloaddition (NOC) reaction for molecular probe generation: a new class of enzyme selective histone deacetylase inhibitors (HDACIs) showing picomolar activity at HDAC6. J. Med. Chem. 51(15), 4370-4373 (2008).

65 Chen Y, Lopez-Sanchez M, Savoy DN, Billadeau DD, Dow GS, Kozikowski AP. A series of potent and selective, triazolylphenyl-based histone deacetylases inhibitors with activity against pancreatic cancer cells and Plasmodium falciparum. J. Med. Chem. 51(12), 3437-3448 (2008).

66 Auzzas L, Larsson A, Matera R et al. Non-natural macrocyclic inhibitors of histone deacetylases: design, synthesis, and activity. J. Med. Chem. 53(23), 8387-8399 (2010).

67 Yang Z, Wang TJ, Wang F et al. Discovery of selective histone deacetylase 6 inhibitors using the quinazoline as the cap for the treatment of cancer. J. Med. Chem. 59(4), 1455-1470 (2016).

68 Fleming CL, Natoli A, Schreuders J et al. Highly fluorescent and HDAC6 selective scriptaid analogues. Eur. J. Med. Chem. 162, 321-333 (2019).

69 Yu CW, Chang PT, Hsin LW, Chern JW. Quinazolin-4-one derivatives as selective histone deacetylase-6 inhibitors for the treatment of Alzheimer's disease. J. Med. Chem. 56(17), 6775-6791 (2013).

70 Lee HY, Tsai AC, Chen MC et al. Azaindolylsulfonamides, with a more selective inhibitory effect on histone deacetylase 6 activity, exhibit antitumor activity in colorectal cancer HCT116 cells. J. Med. Chem. 57(10), 4009-4022 (2014).

71 Wagner FF, Olson DE, Gale JP et al. Potent and selective inhibition of histone deacetylase 6 (HDAC6) does not require a surface-binding motif. J. Med. Chem. 56(4), 1772-1776 (2013).

72 De Vreese R, Van Steen N, Verhaeghe T et al. Synthesis of benzothiophene-based hydroxamic acids as potent and selective HDAC6 inhibitors. Chem. Commun. 51(48), 9868-9871 (2015).

73 Lee HY, Nepali K, Huang FI et al. (N-Hydroxycarbonylbenylamino)quinolines as selective histone deacetylase 6 inhibitors suppress growth of multiple myeloma in vitro and in vivo. J. Med. Chem. 61(3), 905-917 (2018).

74 Kozikowski AP, Shen S, Pardo M et al. Brain penetrable histone deacetylase 6 inhibitor SW-100 ameliorates memory and learning impairments in a mouse model of fragile X syndrome. ACS Chem. Neurosci. 10(3), 1679-1695 (2019).

75 Shen SD, Benoy V, Bergman JK et al. Bicyclic-capped histone deacetylase 6 inhibitors with improved activity in a model of axonal charcot-marie-tooth disease. ACS Chem. Neurosci. 7(2), 240-258 (2016).

76 Vergani B, Sandrone G, Marchini M et al. Novel benzohydroxamate-based potent and selective histone deacetylase 6 (HDAC6) inhibitors bearing a pentaheterocyclic scaffold: design, synthesis, and biological evaluation. J. Med. Chem. 62(23), 10711-10739 (2019).

77 Gawel JM, Shouksmith AE, Raouf YS et al. PTG-0861: A novel HDAC6-selective inhibitor as a therapeutic strategy in acute myeloid leukaemia. Eur. J. Med. Chem. 201, 112411 (2020).

78 Smil DV, Manku S, Chantigny YA et al. Novel HDAC6 isoform selective chiral small molecule histone deacetylase inhibitors. Bioorg. Med. Chem. Lett. 19(3), 688-692 (2009).

79 Liu YM, Lee HY, Lai MJ et al. Pyrimidinedione-mediated selective histone deacetylase 6 inhibitors with antitumor activity in colorectal cancer HCT116 cells. Org. Biomol. Chem. 13(40), 10226-10235 (2015).

80 Liu JR, Yu CW, Hung PY, Hsin LW, Chern JW. High-selective HDAC6 inhibitor promotes HDAC6 degradation following autophagy modulation and enhanced antitumor immunity in glioblastoma. Biochem. Pharmacol. 163, 458-471 (2019).

81 Vishwakarma S, Iyer LR, Muley M et al. Tubastatin, a selective histone deacetylase 6 inhibitor shows anti-inflammatory and anti-rheumatic effects. Int. Immunopharmacol. 16(1), 72-78 (2013).

82 Kozlov MV, Kleymenova AA, Konduktorov KA, Malikova AZ, Kochetkov SN. Selective inhibitor of histone deacetylase 6 (tubastatin A) suppresses proliferation of hepatitis C virus replicon in culture of human hepatocytes. Biochemistry (Moscow) 79(7), 637-642 (2014).

83 Shen SD, Svoboda M, Zhang GM et al. Structural and in vivo characterization of tubastatin a, a widely used histone deacetylase 6 inhibitor. ACS Med. Chem. Lett. 11(5), 706-712 (2020).

84 Sellmer A, Stangl H, Beyer M et al. Marbostat-100 defines a new class of potent and selective antiinflammatory and antirheumatic histone deacetylase 6 inhibitors. J. Med. Chem. 61(8), 3454-3477 (2018).

85 Leonhardt M, Sellmer A, Kramer $\mathrm{OH}$ et al. Design and biological evaluation of tetrahydro-beta-carboline derivatives as highly potent histone deacetylase 6 (HDAC6) inhibitors. Eur. J. Med. Chem. 152, 329-357 (2018).

86 De Vreese R, Depetter Y, Verhaeghe T et al. Synthesis and SAR assessment of novel Tubathian analogs in the pursuit of potent and selective HDAC6 inhibitors. Org. Biomol. Chem. 14(8), 2537-2549 (2016).

87 De Vreese R, Galle L, Depetter Y et al. Synthesis of potent and selective HDAC6 inhibitors bearing a cyclohexane- or cycloheptane-annulated 1,5-benzothiazepine scaffold. Chemistry 23(1), 128-136 (2017).

88 Vögerl K, Ong N, Senger J et al. Synthesis and biological investigation of phenothiazine-based benzhydroxamic acids as selective histone deacetylase 6 inhibitors. J. Med. Chem. 62(3), 1138-1166 (2019). 
89 Lin XF, Chen WM, Qiu ZX et al. Design and synthesis of orally bioavailable aminopyrrolidinone histone deacetylase 6 inhibitors. J. Med. Chem. 58(6), 2809-2820 (2015).

90 Chen X, Chen XY, Steimbach RR et al. Novel 2, 5-diketopiperazine derivatives as potent selective histone deacetylase 6 inhibitors: Rational design, synthesis and antiproliferative activity. Eur. J. Med. Chem. 187, 111950 (2020).

91 Liang T, Hou XB, Zhou Y, Yang XY, Fang H. Design, synthesis, and biological evaluation of 2,4-imidazolinedione derivatives as HDAC6 isoform-selective inhibitors. ACS Med. Chem. Lett. 10(8), 1122-1127 (2019).

92 Bergman JA, Woan K, Perez-Villarroel P, Villagra A, Sotomayor EM, Kozikowski AP. Selective histone deacetylase 6 inhibitors bearing substituted urea linkers inhibit melanoma cell growth. J. Med. Chem. 55(22), 9891-9899 (2012).

93 Lee JH, Mahendran A, Yao YS et al. Development of a histone deacetylase 6 inhibitor and its biological effects. Proc. Natl Acad. Sci. USA 110(39), 15704-15709 (2013).

94 Jochems J, Boulden J, Lee BG et al. Antidepressant-like properties of novel HDAC6-selective inhibitors with improved brain bioavailability. Neuropsychopharmacology 39(2), 389-400 (2014).

95 Krukowski K, Ma J, Golonzhka O et al. HDAC6 inhibition effectively reverses chemotherapy-induced peripheral neuropathy. Pain 158(6), 1126-1137 (2017).

96 Benoy V, Vanden Berghe P, Jarpe M, Van Damme P, Robberecht W, Van Den Bosch L. Development of improved HDAC6 inhibitors as pharmacological therapy for axonal charcot-marie-tooth disease. Neurotherapeutics 14(2), 417-428 (2017).

97 Senger J, Melesina J, Marek M et al. Synthesis and biological investigation of oxazole hydroxamates as highly selective histone deacetylase 6 (HDAC6) inhibitors. J. Med. Chem. 59(4), 1545-1555 (2016).

98 Shen SD, Hadley M, Ustinova K et al. Discovery of a new isoxazole-3-hydroxamate-based histone deacetylase 6 inhibitor SS-208 with antitumor activity in syngeneic melanoma mouse models. J. Med. Chem. 62(18), 8557-8577 (2019).

99 Blackburn C, Barrett C, Chin J et al. Potent histone deacetylase inhibitors derived from 4-(aminomethyl)-N-hydroxybenzamide with high selectivity for the HDAC6 isoform. J. Med. Chem. 56(18), 7201-7211 (2013).

100 Blackburn C, Barrett C, Brunson M et al. Histone deacetylase inhibitors derived from 1,2,3,4-tetrahydropyrrolo[1,2-a] pyrazine and related heterocycles selective for the HDAC6 isoform. Bioorg. Med. Chem. Lett. 24(23), 5450-5454 (2014).

101 Song Y, Lim J, Seo YH. A novel class of anthraquinone-based HDAC6 inhibitors. Eur. J. Med. Chem. 164, 263-272 (2019).

102 Strebl MG, Campbell AJ, Zhao WN et al. HDAC6 brain mapping with [(18)F]bavarostat enabled by a ru-mediated deoxyfluorination. ACS Cent. Sci. 3(9), 1006-1014 (2017).

103 Kozikowski AP, Chen YF, Gaysin A et al. Functional differences in epigenetic modulators - superiority of mercaptoacetamide-based histone deacetylase inhibitors relative to hydroxamates in cortical neuron neuroprotection studies. J. Med. Chem. 50(13), 3054-3061 (2007).

104 Kalin JH, Zhang H, Gaudrel-Grosay S, Vistoli G, Kozikowski AP. Chiral mercaptoacetamides display enantioselective inhibition of histone deacetylase 6 and exhibit neuroprotection in cortical neuron models of oxidative stress. ChemMedChem 7(3), 425-439 (2012).

105 Patil V, Sodji QH, Kornacki JR, Mrksich M, Oyelere AK. 3-Hydroxypyridin-2-thione as novel zinc binding group for selective histone deacetylase inhibition. J. Med. Chem. 56(9), 3492-3506 (2013).

106 Sodji QH, Patil V, Kornacki JR, Mrksich M, Oyelere AK. Synthesis and structure-activity relationship of 3-hydroxypyridine-2-thione-based histone deacetylase inhibitors. J. Med. Chem. 56(24), 9969-9981 (2013).

107 Muthyala R, Shin WS, Xie JS, Sham YY. Discovery of 1-hydroxypyridine-2-thiones as selective histone deacetylase inhibitors and their potential application for treating leukemia. Bioorg. Med. Chem. Lett. 25(19), 4320-4324 (2015).

108 Seidel C, Schnekenburger M, Mazumder A et al. 4-Hydroxybenzoic acid derivatives as HDAC6-specific inhibitors modulating microtubular structure and HSP90 alpha chaperone activity against prostate cancer. Biochem. Pharmacol. 99, 31-52 (2016).

109 Liu T, Wan Y, Xiao Y, Xia C, Duan G. Dual-target inhibitors based on HDACs: novel antitumor agents for cancer therapy. J. Med. Chem. 63(17), 8977-9002 (2020).

- Introduces a theoretical basis for designing HDAC-involved dual-target inhibitors and providing insight into the structure-activity relationship of these agents.

110 Hesham HM, Lasheen DS, Abouzid KaM. Chimeric HDAC inhibitors: comprehensive review on the HDAC-based strategies developed to combat cancer. Med. Res. Rev. 38(6), 2058-2109 (2018).

111 Li Y, Seto E. HDACs and HDAC inhibitors in cancer development and therapy. Cold Spring Harb. Perspect. Med. 6(10), a026831 (2016).

112 Vanhaesebroeck B, Leevers SJ, Panayotou G, Waterfield MD. Phosphoinositide 3-kinases: a conserved family of signal transducers. Trends Biochem. Sci. 22(7), 267-272 (1997).

113 Thakur A, Tawa GJ, Henderson MJ et al. Design, synthesis, and biological evaluation of quinazolin-4-one-based hydroxamic acids as dual PI3K/HDAC inhibitors. J. Med. Chem. 63(8), 4256-4292 (2020).

114 O'shea JJ, Plenge R. JAK and STAT signaling molecules in immunoregulation and immune-mediated disease. Immunity 36(4), 542-550 (2012). 
115 Bhagwat N, Levine RL, Koppikar P. Sensitivity and resistance of JAK2 inhibitors to myeloproliferative neoplasms. Int. J. Hematol. 97(6), 695-702 (2013).

116 Yang EG, Mustafa N, Tan EC et al. Design and synthesis of janus kinase 2 (JAK2) and histone deacetlyase (HDAC) bispecific inhibitors based on pacritinib and evidence of dual pathway inhibition in hematological cell lines. J. Med. Chem. 59(18), 8233-8262 (2016).

117 Huang YH, Dong GQ, Li HQ, Liu N, Zhang WN, Sheng CQ. Discovery of janus kinase 2 (JAK2) and histone deacetylase (HDAC) dual inhibitors as a novel strategy for the combinational treatment of leukemia and invasive fungal infections. J. Med. Chem. 61(14), 6056-6074 (2018).

118 Yao LB, Ramanujulu PM, Poulsen A, Ohlson S, Dymock BW. Merging of ruxolitinib and vorinostat leads to highly potent inhibitors of JAK2 and histone deacetylase 6 (HDAC6). Bioorg. Med. Chem. Lett. 28(15), 2636-2640 (2018).

119 Liang XW, Zang J, Li XY et al. Discovery of novel janus kinase (JAK) and histone deacetylase (HDAC) dual inhibitors for the treatment of hematological malignancies. J. Med. Chem. 62(8), 3898-3923 (2019).

120 Pei X-Y, Dai Y, Grant S. Synergistic induction of oxidative injury and apoptosis in human multiple myeloma cells by the proteasome inhibitor bortezomib and histone deacetylase inhibitors. Clin. Cancer Res. 10(11), 3839-3852 (2004).

121 Bhatia S, Krieger V, Groll M et al. Discovery of the first-in-class dual histone deacetylase-proteasome inhibitor. J. Med. Chem. 61(22), 10299-10309 (2018).

122 Baillie GS, Tejeda GS, Kelly MP. Therapeutic targeting of 3', 5'-cyclic nucleotide phosphodiesterases: inhibition and beyond. Nat. Rev. Drug Discov. 18(10), 770-796 (2019).

123 Rabal O, Sanchez-Arias JA, Cuadrado-Tejedor M et al. Design, synthesis, biological evaluation and in vivo testing of dual phosphodiesterase 5 (PDE5) and histone deacetylase 6 (HDAC6)-selective inhibitors for the treatment of Alzheimer's disease. Eur. J. Med. Chem. 150, 506-524 (2018).

124 Rabal O, Sanchez-Arias JA, Cuadrado-Tejedor M et al. Multitarget approach for the treatment of Alzheimer's disease: inhibition of phosphodiesterase 9 (PDE9) and histone deacetylases (HDACs) covering diverse selectivity profiles. ACS Chem. Neurosci. 10(9), 4076-4101 (2019).

125 Cuadrado-Tejedor M, Pérez González M, García-Muñoz C et al. Taking advantage of the selectivity of histone deacetylases and phosphodiesterase inhibitors to design better therapeutic strategies to treat Alzheimer's disease. Front. Aging Neurosci. 11, 149 (2019).

126 Knutson SK, Wigle TJ, Warholic NM et al. A selective inhibitor of EZH2 blocks H3K27 methylation and kills mutant lymphoma cells. Nat. Chem. Biol. 8(11), 890-896 (2012).

127 Yamaguchi J, Sasaki M, Sato Y et al. Histone deacetylase inhibitor (SAHA) and repression of EZH2 synergistically inhibit proliferation of gallbladder carcinoma. Cancer Sci. 101(2), 355-362 (2010).

128 Romanelli A, Stazi G, Fioravanti R et al. Design of first-in-class dual EZH2/HDAC inhibitor: biochemical activity and biological evaluation in cancer cells. ACS Med. Chem. Lett. 11(5), 977-983 (2020).

129 Schopf FH, Biebl MM, Buchner J. The HSP90 chaperone machinery. Nat. Rev. Mol. Cell Biol. 18(6), 345 (2017).

130 Ojha R, Nepali K, Chen CH et al. Isoindoline scaffold-based dual inhibitors of HDAC6 and HSP90 suppressing the growth of lung cancer in vitro and in vivo. Eur. J. Med. Chem. 190, 112086 (2020).

131 Pinzi L, Benedetti R, Altucci L, Rastelli G. Design of dual inhibitors of histone deacetylase 6 and heat shock protein 90. ACS Omega 5(20), 11473-11480 (2020).

132 Choudhari SK, Chaudhary M, Bagde S, Gadbail AR, Joshi V. Nitric oxide and cancer: a review. World J. Surg. Onc. 11, 118 (2013).

133 Duan WW, Li J, Inks ES et al. Design, synthesis, and antitumor evaluation of novel histone deacetylase inhibitors equipped with a phenylsulfonylfuroxan module as a nitric oxide donor. J. Med. Chem. 58(10), 4325-4338 (2015).

134 Zhang X, Liu X, Zhou D, Zheng G. Targeting anti-apoptotic BCL-2 family proteins for cancer treatment. Future Med. Chem. 12(7), 563-565 (2020).

135 Zhou R, Fang S, Zhang M et al. Design, synthesis, and bioactivity evaluation of novel Bcl-2/HDAC dual-target inhibitors for the treatment of multiple myeloma. Bioorg. Med. Chem. Lett. 29(3), 349-352 (2019).

136 Soumyanarayanan U, Ramanujulu PM, Mustafa N et al. Discovery of a potent histone deacetylase (HDAC) $3 / 6$ selective dual inhibitor. Eur. J. Med. Chem. 184, 111755 (2019).

137 Zou Y, Ma D, Wang Y. The PROTAC technology in drug development. Cell Biochem. Funct. 37(1), 21-30 (2019).

138 Guha M. HDAC inhibitors still need a home run, despite recent approval. Nat. Rev. Drug Discov. 14(4), 225-226 (2015).

139 Gryder BE, Sodji QH, Oyelere AK. Targeted cancer therapy: giving histone deacetylase inhibitors all they need to succeed. Future Med. Chem. 4(4), 505-524 (2012).

140 Gryder BE, Wu L, Woldemichael GM et al. Chemical genomics reveals histone deacetylases are required for core regulatory transcription. Nat. Commun. 10(1), 3004 (2019).

141 Zhang X, Thummuri D, He Y et al. Utilizing PROTAC technology to address the on-target platelet toxicity associated with inhibition of BCL-XL. Chem. Commun. 55(98), 14765-14768 (2019). 
142 Smalley JP, Adams GE, Millard CJ et al. PROTAC-mediated degradation of class i histone deacetylase enzymes in corepressor complexes. Chem. Commun. 56(32), 4476-4479 (2020).

143 Roatsch M, Vogelmann A, Herp D, Jung M, Olsen CA. Proteolysis-targeting chimeras (PROTACs) based on macrocyclic tetrapeptides selectively degrade class I histone deacetylases 1-3. ChemRxiv doi: 10.26434/chemrxiv.12416303.v1 (2020).

144 Xiao Y, Wang J, Zhao LY et al. Discovery of histone deacetylase 3 (HDAC3)-specific PROTACs. Chem. Commun. 56(68), 9866-9869 (2020).

145 Gadd MS, Testa A, Lucas X et al. Structural basis of PROTAC cooperative recognition for selective protein degradation. Nat. Chem. Biol. 13(5), 514-521 (2017).

146 Smith BE, Wang SL, Jaime-Figueroa S et al. Differential PROTAC substrate specificity dictated by orientation of recruited E3 ligase. Nat. Commun. 10(1), 131 (2019).

147 Khan S, Zhang X, Lv D et al. A selective BCL-XL PROTAC degrader achieves safe and potent antitumor activity. Nat. Med. 25(12), 1938-1947 (2019).

148 Krönke J, Udeshi ND, Narla A et al. Lenalidomide causes selective degradation of IKZF1 and IKZF3 in multiple myeloma cells. Science 343(6168), 301-305 (2014).

149 Hideshima T, Cottini F, Ohguchi $\mathrm{H}$ et al. Rational combination treatment with histone deacetylase inhibitors and immunomodulatory drugs in multiple myeloma. Blood Cancer J. 5(5), e312-e312 (2015).

150 Ruijter AJMD, Gennip AHV, Caron HN, Kemp S, Kuilenburg ABPV. Histone deacetylases (HDACs): characterization of the classical HDAC family. Biochem. J. 370(3), 737-749 (2003). 
